\title{
Computing continuous-time growth models with boundary conditions via wavelets
}

\author{
Mercedes Esteban-Bravo*, Jose M. Vidal-Sanz
}

Department of Business, Universidad Carlos III de Madrid, C/Madrid no 126, 28903 Getafe, Madrid, Spain

\begin{abstract}
This paper presents an algorithm for solving boundary value differential equations, which often arise in economics from the application of Pontryagin's maximum principle. We propose a wavelet-collocation algorithm, study its convergence properties and illustrate how this approach can be applied to different economic problems.
\end{abstract}

JEL classification: $\mathrm{C} 63$

Keywords: Wavelets; Continuous-time growth models; Boundary value problems

\section{Introduction}

Many economic problems with finite time horizon have the form of boundary value problems $(B V P)$ for differential equations, resulting from Pontryagin's maximum principle. In rare cases the solution can be obtained analytically, but in general, numerical approximation methods are required. Despite the rapid growth on numerical methods for approximating solutions to continuous-time models (for recent surveys see Rust, 1996, Santos, 1999, the text by Judd, 1998 and the collection

\footnotetext{
*Corresponding author.

E-mail addresses: mesteban@emp.uc3m.es (M. Esteban-Bravo),jvidal@emp.uc3m.es (J.M. Vidal-Sanz).
} 
of essays edited by Marimon and Scott, 1999), few algorithms have been developed to cope with boundary conditions (see, e.g. Goffe, 1993).

Given a continuous function $f: \mathbb{R}^{R+1} \rightarrow \mathbb{R}^{R}$, and a vector of continuous linear functionals $\alpha \quad\left(\alpha_{1}, \ldots, \alpha_{R}\right)^{\prime}$, with $\alpha_{j}: C^{1}([a, b])^{R} \rightarrow \mathbb{R}$ linearly independent, consider the $B V P$

$$
\begin{aligned}
& D y(t) \quad f(t, y), \\
& \alpha(y) \quad c,
\end{aligned}
$$

where $y \quad\left(y_{1}, \ldots, y_{R}\right)^{\prime}, \quad y_{j} \in C^{1}([a, b])$ for $j \quad 1, \ldots, R$, and $D y$ denotes the differential operator. The boundary condition specification $\alpha(y) \quad c$ includes most of the initial and $B V P$ considered in macroeconomic analysis. In particular, we are concerned with the boundary conditions of the form $y(t) \quad 0$ for some $t \in[a, b]$, or other more general specifications such as $\alpha(y) \quad \sum_{k}^{K}{ }_{1} A_{k} y\left(t_{k}\right)$, where $A_{k}$ is an $R$ dimensional square matrix and $t_{k} \in[a, b]$, or integral conditions where $\alpha(y) \quad \int A(s) y(s) \mathrm{d} s$.

Several different algorithms have been proposed in numerical analysis literature. Shooting methods are probably the most popular method for solving $B V P$. A shooting method is a successive substitution method based on the idea of guessing the initial condition which associate solution satisfies the desired boundary condition. Then, any finite difference algorithm can be considered to solve this 'new' initial value problem. For details see, e.g. Ascher et al. (1995), Roberts and Shipman (1972) and Keller (1976). Unfortunately, these methods can be quite inefficient as they may often converge quite slowly, or not at all, and a wrong guess could substantially increase the computer time. Furthermore, the numerical errors can be magnified. The possible difficulties with shooting methods are frequently discussed in the literature, see, e.g. Conte (1966), Keller $(1968,1976)$, and Osborne (1969).

Alternatively, $B V P$ can be solved using some projection based methods, such as Galerkin or collocation techniques. Those based on splines are commonly used and their theory is well developed in numerical analysis (see, e.g. Varga, 1971; Russell and Shampine, 1972; Lucas and Reddien, 1972; de Boor and Schwartz, 1973; Prenter, 1975). In this context collocation methods often have better performance than Galerkin methods, but the choice of the collocation points greatly influences the effectiveness of the method. Furthermore, if the solution path exhibits some abrupt changes, the approximation could be inaccurate.

The use of wavelets in the projection methods is superficially similar to other bases. Wavelet bases have the attractive property that once one of the basis functions is known the rest may be obtained by dilation and integer translation of a single function. Wavelets have been applied to a wide range of problems such as signal processing, image analysis, data compression and time series econometrics. Particularly, the discovery of compactly supported wavelets has proven to be a useful tool for the approximation of functions, differential and integral operators. The Daubechies wavelets that we use in this paper form an orthogonal basis with compact support. A short support is a desirable property because this makes the 
approximation analysis local. These and other features are that they allow us to treat a large class of operators in an efficient way (as documented in, e.g. Beylkin, 1992, 1993).

In this paper we propose a methodology for approximating the solution of $B V P$. Given a wavelets basis, we approximate the solution of the trapezoidal discretization of the $B V P$. The use of trapezoidal discretization avoids the numerical instabilities often observed in many algorithms for solving ordinary differential equations (e.g. the Euler finite approximation for stiff problems). For approximating the solution to models in which inequality constraints occasionally bind, we present an extension of the proposed algorithm that deals appropriately with inequality constraints.

The rest of the paper is organized as follows. Section 2 contains an introduction to wavelets and the Daubechies wavelets that we use in this paper are presented in detail. Section 3 describes a wavelet collocation for solving a $B V P$ and some examples that illustrate the good performance of the algorithm (Appendix A presents a MATLAB code for solving a simple example). Section 4 presents the theoretical convergence analysis. All the proofs can be found in Appendix B.

\section{An introduction to wavelets}

The proposed algorithm for solving $B V P$ relies heavily on wavelets approximation theory. Thus, before presenting the wavelet collocation approach, let us introduce wavelets in some detail.

Let $L_{2}(\mathbb{R})$ denote the vector space of all classes of Lebesgue measurable functions $y$ defined on $\mathbb{R}$ (we identify functions that are equal almost everywhere) such that $\int|y(t)|^{2} \mathrm{~d} t<\infty$, which is a Hilbert space endowed with the inner product $\langle x, y\rangle_{L_{2}} \quad \int x(t) \overline{y(t)} \mathrm{d} t$.

Consider a sequence of closed subspaces $\left\{V_{n}\right\}_{n \in \mathbb{Z}}$ of $L_{2}(\mathbb{R})$ such that $V_{n} \subset V_{n+1}$, for all $n \in \mathbb{Z}, \bigcap_{n \in \mathbb{Z}} V_{n} \quad\{0\}$, and $\bigcup_{n \in \mathbb{Z}} V_{n}$ is dense in $L_{2}(\mathbb{R})$. We say that $\left\{V_{n}\right\}_{n \in \mathbb{Z}}$ is a multiresolution if it satisfies the conditions

(i) $x(t) \in V_{n} \Leftrightarrow x(2 t) \in V_{n+1}$.

(ii) $x(t) \in V_{0} \Leftrightarrow x(t+1) \in V_{0}$.

(iii) There exists a function $\phi \in V_{0}$, known as the father wavelet or scaling function, with a nonvanishing integral and such that $\{\phi(t \quad k)\}_{k \in \mathbb{Z}}$ is an orthonormal basis of $V_{0}$. (Actually, it is sufficient that $\left\{\phi\left(\begin{array}{ll}t & k\end{array}\right)\right\}_{k \in \mathbb{Z}}$ form a Riesz basis ${ }^{1}$ ).

The multiresolution concept was introduced by Mallat (1989). If $\left\{V_{n}\right\}$ is a multiresolution, then each subspace $V_{n}$ is the span of an orthonormal basis $\left\{\phi_{n, k}\right\}_{k \in \mathbb{Z}}$, with $\phi_{n, k}(t) \quad 2^{n / 2} \phi\left(2^{n} t \quad k\right)$.

Wavelet theory considers the representation of general functions in terms of simpler, fixed building blocks $\phi_{n, k}$ at different scales and positions. As the set of

\footnotetext{
${ }^{1}$ A countable set $\left\{f_{n}\right\}$ of a Hilbert space is a Riesz Basis if every element $y$ of the space can be written uniquely as $f=\sum_{n} c_{n} f_{n}$, and there exists positive constants $A, B$ such that $A\|f\|^{2}<\sum_{n}\left|c_{n}\right|^{2}<B\|f\|^{2}$. Orthonormal and biorthogonal basis are particular cases.
} 
functions $\left\{\phi\left(\begin{array}{ll}t & k\end{array}\right)\right\}_{k \in \mathbb{Z}}$ is orthonormal, the orthogonal projection of an arbitrary $y \in L_{2}(\mathbb{R})$ into $V_{n}$ can be written as $\Pi_{V_{n}}(y)(t) \quad \sum_{k \in \mathbb{Z}}\left\langle y, \phi_{n, k}\right\rangle_{L_{2}} \phi_{n, k}(t)$, yielding the best $L_{2}$ approximation of the function $y$ in $V_{n}$, and furthermore

$$
y(t) \quad \lim _{n \rightarrow \infty} \Pi_{V_{n}}(y)(t) \quad \lim _{n \rightarrow \infty} \sum_{k \in \mathbb{Z}}\left\langle y, \phi_{n, k}\right\rangle_{L_{2}} \phi_{n, k}(t),
$$

i.e. $\left\|y \quad \Pi_{V_{n}}(y)\right\| \rightarrow 0$ in the sense of $L_{2}$. For each $t \in \mathbb{R}$ the summation in (2) contains a finite number of nonnull terms whenever $\phi$ has compact support, otherwise it should be truncated for practical applications. Under appropriate conditions, the approximation property holds in the supremum norm, for continuous functions $y(t)$ with compact support. The wavelet multiresolution can be analogously defined on $L_{2}([a, b])$, considering subspaces $\left\{V_{n}\right\}_{n 1}^{\infty}$ and $\phi$ supported on $[a, b]$.

Each wavelet system contains functions $\phi_{n, k}$ that vary in time and frequency. Wavelets decompose functions in components with different frequencies, and then study each component with a resolution matched to its scale. The efficiency of a wavelet system approximating functions depends on the Fourier transform of the scaling $\Phi(\omega) \quad \int \phi(t) \mathrm{e}^{-\mathrm{i} \omega t} \mathrm{~d} t$. For example, the speed to which $\left\|y \quad \Pi_{V_{n}}(y)\right\| \rightarrow 0$ for functions $y$ with several continuous derivatives is typically higher when the scaling satisfies $\left.D^{j} \Phi(\omega)\right|_{\omega} 0 \quad 0$ for $j \quad 1, \ldots, q$ for a larger $q$. This property is known as the vanishing moments property, since it is equivalent to $\int \phi(u) u^{j} \mathrm{~d} u$ ofor $j \quad 1, \ldots, q$ (i.e. the basis functions are chosen to be orthogonal to the lower degree polynomials). Note also that $\{\phi(t \quad k)\}_{k \in \mathbb{Z}}$ is an orthonormal basis when $\sum_{k \in \mathbb{Z}}|\Phi(\omega+2 \pi k)|^{2} \quad 1$ a.e. Lemarié (1988) proposed an orthogonalization proce dure to find orthonormal wavelets. If the integer translations of $\phi$ form a Riesz basis of $V_{0}$, then an orthonormal basis can be defined by $\phi^{\text {orth }}$ and its integer translations, where $\Phi^{\text {orth }}(\omega) \quad \Phi(\omega) / \sqrt{ } \sum_{k \in \mathbb{Z}}|\Phi(\omega+2 \pi k)|^{2}$. But if $\phi$ is compactly supported, $\phi^{\text {orth }}$ will not be so in general.

Wavelets are well suited for approximating functions with discontinuities and sharp spikes. In order to approximate functions with isolate discontinuities, one would like basis with a very short support. At the same time, to obtain a detailed frequency analysis (a good representation of small bumps and cycles) it is preferable that the support of the Fourier transform $\Phi$ is a short interval (which means that $\phi$ is smooth). This implies that the support of $\phi$ cannot be short (the Heisenberg Uncertainty Principle establishes that functions cannot be frequency band limited and time limited simultaneously). Different wavelet families make a different trade off between how compactly the basis functions are localized in time (how well rough functions are approximated) and frequency (how smooth they are).

Many wavelet families have been considered in the literature. The simplest father wavelet is the Haar wavelet

$$
\phi_{1}(t) \quad I_{[0,1)}(t),
$$


where $I_{A}(t) \quad 1$ if $t \in A$ and zero otherwise. Another classical example is the Shannon function

$$
\phi^{s}(t) \quad \sin (\pi t) / \pi t,
$$

with Fourier transform $\Phi^{s}(\omega) \quad I_{[-\pi, \pi)}(\omega)$. Haar and Shannon wavelets are extreme opposite cases, regarding their efficacy in the time and frequency domain. The first scaling function has very low regularity and the second a very slow decay in the time domain, and reciprocally in the frequency. Haar wavelets can be generalized to the cardinal $B$ spline biorthogonal wavelets of order $N$, defined recursively as a convolution $\phi_{N}(t) \quad \phi_{N-1}(t) * \phi_{1}(t)$, with $\phi_{1}$ the Haar wavelet. The Fourier transform of $\phi_{N}$ is $\Phi_{N}(\omega) \quad\left(\left(1 \quad \mathrm{e}^{-\mathrm{i} \omega}\right) / \mathrm{i} \omega\right)^{N}$. These bases are not orthogonal (orthogonality means no redundancy in the representation and hence, it is not required unnecessary computer time or storage). When the Lemariés orthogonaliza tion process is applied to the B splines biorthogonal wavelets, we obtain the Battle Lemarié wavelets (see, e.g. Battle, 1987; Lemarié, 1988). The Battle Lemarié scaling functions do not have compact support but have an exponential decay.

Probably, the most frequently used wavelets systems are the compactly supported wavelets proposed by Daubechies (1992). Daubechies' wavelets are indexed by $N \in\{1,2,3, \ldots\}$, which is the number of vanishing moments. Due to the fact that $V_{0} \subset V_{1}$, any father wavelet can be expressed as

$$
\phi(t) \quad \sum_{k \in \mathbb{Z}} a_{k} \phi(2 t \quad k),
$$

for some $\left\{a_{k}\right\}_{k \in \mathbb{Z}} \in l_{2}$. Taking Fourier transforms, we can express (3) as

$$
\Phi(\omega) \quad A(\omega / 2) \Phi(\omega / 2),
$$

where $A(\omega) \quad(1 / 2) \sum_{k \in \mathbb{Z}} a_{k} \mathrm{e}^{-\mathrm{i} k \omega}$. A multiresolution can be defined by finding a function $A(\omega)$, which means finding a sequence $\left\{a_{k}\right\}$ such that the Fourier inverse of $\Phi$ satisfying (4) is a father wavelet. Daubechies proposed a procedure to construct a finite sequence $\left\{a_{k}\right\}_{k}^{2 N-1}$ such that

$$
\phi(t) \quad \sum_{k=1}^{2 N-1} a_{k} \phi(2 t \quad k),
$$

and the resulting $\phi$ has compact support with length $2 N \quad 1$. From the computational point of view, a high number of vanishing moments $N$ translates into a sparse representation (2) of a piecewise smooth function $y(t)$, as the wavelet coefficients $\left\langle y, \phi_{n, k}\right\rangle_{L_{2}}$ will be zero when $2^{n / 2} \phi\left(2^{n} t \quad k\right)$ is supported on regions where $y$ is well approximated by an $N \quad 1$ degree polynomial. The support for the scaling function $\phi_{n, k}$ is $\left[2^{-n} k, 2^{-n}(k+2 N \quad 1)\right]$. Then, the higher the $N$ the longer the support of $\phi$ and this effect reduces the localization, increasing the number of functions $\phi_{n, k}(t)$ required to approximate $y$ at $t$ for a particular $n$. For $N>1$ the Daubechies wavelets do not have analytic expression $(N \quad 1$ leads to the Haar wavelets), but can be computed by a recursive algorithm known as the cascade algorithm. Figs. 1 and 2 present Daubechies scaling functions of order $N \quad 3$ and 4, respectively, where it can be observed the support enlargement with the order $N$. For 


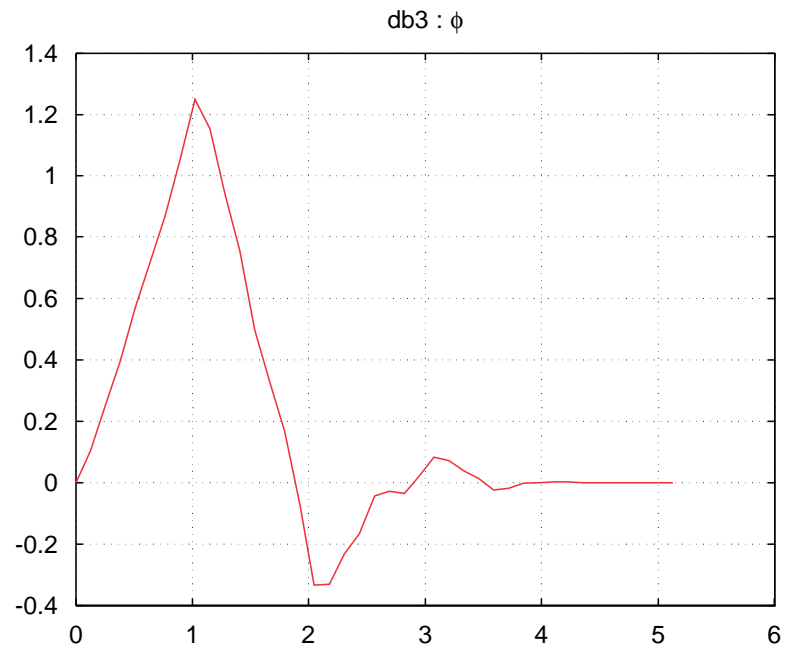

Fig. 1. Daubechies scaling function of order $N=3$.

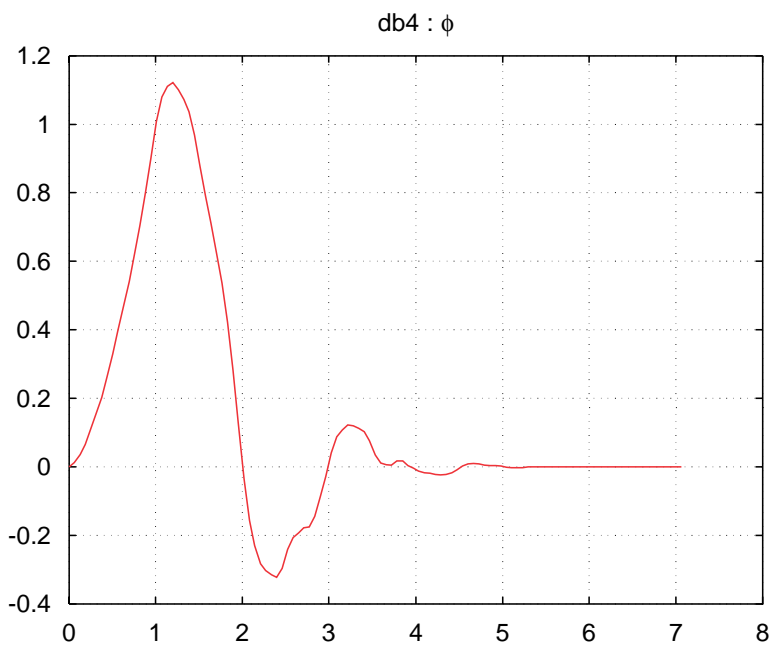

Fig. 2. Daubechies scaling function of order $N=4$.

a detailed exposition about Daubechies wavelets of order $N$ and their properties, see Daubechies (1992). Daubechies wavelets have been adapted to suit specific requirements such as symmetry (called symlets) or subspaces such as $L_{2}([a, b])$ (see Daubechies, 1994). 
Let $W_{2}^{r}(\mathbb{R})$ be the Sobolev space in $L_{2}$ (i.e. the $L_{2}$ closure of $C^{r}(\mathbb{R})$ ). The elements of $W_{2}^{r}(\mathbb{R})$ are all the functions weakly differentiable ${ }^{2}$ up to order $r$ with weak derivatives square integrable. Therefore, $D^{m} y$ can be approximated by $\sum_{k \in \mathbb{Z}}\left\langle D^{m} y, \phi_{n, k}\right\rangle_{L_{2}} \phi_{n, k}$ when $n \rightarrow \infty$. Some wavelet bases have a scaling function $\phi$ in the Sobolev space (for example, the Daubechies wavelets of order $N$ satisfy $\left.\phi \in W_{2}^{N}(\mathbb{R})\right)$, which can be used to approximate the derivatives of smooth functions $y \in W_{2}^{r}(\mathbb{R})$, as follows:

$$
D^{m} y(t) \quad \lim _{n \rightarrow \infty} \sum_{k \in \mathbb{Z}}\left\langle y, \phi_{n, k}\right\rangle_{L_{2}} D^{m} \phi_{n, k}(t),
$$

for any derivative of order $m \leqslant N$. This convergence is uniform if $y \in C^{r}(\mathbb{R})$ and has compact support (Daubechies wavelets have a scaling function $\phi \in C^{N}(\mathbb{R})$ ). As $D^{m} \phi \in L_{2}\left(\mathbb{R}^{d}\right)$, we can also express the derivative $D^{m} \phi_{n, k}$ in terms of the wavelet basis,

$$
D^{m} \phi_{n, k}(t) \quad \lim _{s \rightarrow \infty} \sum_{r \in \mathbb{Z}}\left\langle D^{m} \phi_{n, k}, \phi_{s, r}\right\rangle_{L_{2}} \phi_{s, r}(t) \quad \lim _{s \rightarrow \infty} \sum_{r \in \mathbb{Z}} \Gamma_{n, k, s, r}^{m} \phi_{s, r}(t),
$$

where the coefficients $\Gamma_{n, k, s, r}^{m}$, known as the connection coefficients, have been computed for the most commonly used wavelets (see Latto et al., 1991 and Beylkin, 1992). Therefore, the derivatives of $y \in W_{2}^{r}(\mathbb{R})$ can be expressed as

$$
D^{m} y(t) \quad \lim _{n, s \rightarrow \infty} \sum_{r \in \mathbb{Z}} \sum_{k \in \mathbb{Z}}\left\langle y, \phi_{n, k}\right\rangle_{L_{2}} \Gamma_{n, k, s, r}^{m} \phi_{s, r}(t) .
$$

However, we avoid the numerical computation of derivatives to reduce the computational cost and to increase the numerical stability in the numerical algorithm presented later in this paper.

Wavelet representations have additional nice properties. For example, given the multiresolution $\left\{V_{n}\right\}$ we can define a Wold type decomposition of $L_{2}\left(\mathbb{R}^{d}\right)$, i.e. a sequence of linear subspaces $\left\{W_{n}\right\}_{n \in \mathbb{Z}}$, such that $W_{n} \perp W_{m}$ if $n \neq m, V_{n+1}$ $V_{n} \oplus W_{n}$ (this requires that $\left.V_{n} \perp W_{n}\right)$ and $\oplus_{n \in \mathbb{Z}} W_{n}$ is dense in $L_{2}\left(\mathbb{R}^{d}\right)$. The subspace $W_{n}$ is called the detail space at level $n$. The sequence $\left\{W_{n}\right\}$ characterizes the multiresolution $\left\{V_{n}\right\}$ and vice versa, as $V_{n} \oplus_{j-\infty}^{n} W_{j}$ and $W_{n} \quad V_{n+1} \ominus V_{n}$. There exists a function $\psi \in L_{2}\left(\mathbb{R}^{d}\right)$, called mother wavelet, such that $W_{n} \quad \operatorname{span}\left\{2^{n d / 2} \psi\left(2^{n} t \quad k\right): k \in \mathbb{Z}^{d}\right\}$, and we can express any $y \in L_{2}\left(\mathbb{R}^{d}\right)$ as

$$
y(t) \quad \lim _{N \rightarrow \infty} \sum_{n}^{N} \Pi_{W_{n}}(y) \quad \sum_{n \in \mathbb{Z}} \sum_{k \in \mathbb{Z}}\left\langle y, \psi_{n, k}\right\rangle_{L_{2}} \psi_{n, k}(t) .
$$

\footnotetext{
${ }^{2}$ We say that a Lebesgue-measurable function $f$ is weakly differentiable if it is integrable on compact subsets and there exists a function $D f$, known as the weak derivative of $f$, such that $\int \phi \cdot f=\int(\phi \cdot D f)$ for all smooth functions $\phi$ with compact support.
} 
Moreover, as $V_{n+k} \quad V_{n} \oplus_{j 1_{1}}^{k} W_{n+j}$ and $V_{0} \quad \oplus_{n-\infty}^{0} W_{n}$, the subspace $V_{0} \oplus\left(\oplus_{n}^{\infty}{ }_{1} W_{n}\right)$ is dense in $L_{2}\left(\mathbb{R}^{d}\right)$, and we can express

$$
\begin{array}{ll}
y(t) \quad & \Pi_{V_{0}}(y)+\lim _{N \rightarrow \infty} \sum_{n 1}^{N} \Pi_{W_{n}}(y) \\
& \sum_{k \in \mathbb{Z}}\left\langle y, \phi_{0, k}\right\rangle_{L_{2}} \phi_{0, k}(t)+\sum_{n=1}^{\infty} \sum_{k \in \mathbb{Z}}\left\langle y, \psi_{n, k}\right\rangle_{L_{2}} \psi_{n, k}(t) .
\end{array}
$$

At the request of R. Coifman, Daubechies developed a variation of her basis (called coiflets) to have both scaling and mother functions with vanishing moments, for details, see Daubechies (1992).

The first wavelet basis can be at least traced to Haar's work (1910), but the theoretical foundations of wavelets were established by physicists and mathemati cians from the early 1930s to the 1980s. The interest in wavelets has increased since Mallat (1989) and Meyer (1992) introduced the use of multiresolution as a framework to study wavelet expansions. A historical perspective can be found in Daubechies (1992) and Meyer (1993). Excellent monographs in wavelets are Chui (1992), Daubechies (1992), Meyer (1992, 1993) and Walnut (2001).

While wavelets may seem 'just another basis', nonetheless they offer considerable advantages. Beylkin (1992, 1993) established the efficiency of wavelets for solving ordinary differential equations. The Laplace operator is diagonally dominant with respect to appropriate wavelet basis, and the matrices associated to this operator are usually sparse. As a consequence, the number of iterations required for solving large classes of ordinary differential equations is fairly small by contrast to other bases. Furthermore, the accuracy of the solution is controlled by a single parameter $n$, and different resolutions can be used in different regions of space. This is particularly useful if the solution of the differential equation is rapidly varying in a particular time region, and the coupling between different resolution levels is simple.

Finally, notice that a large number of software sources is available. For example, MATLAB has a toolbox specialized in wavelets. In addition, WaveLab is a collection of MATLAB toolboxes (libraries) available from Stanford University that can be found at http://www stat.stanford.edu/ wavelab/. The Computational Mathematics Laboratory has made available another MATLAB wavelet toolbox that can be found at http://www dsp.rice.edu/software/.

\section{The wavelet-collocation method}

In this section we describe a wavelet collocation algorithm for solving $B V P(1)$.

The first step of the proposed method is to consider the best approximation of $y$. For the sake of convenience, let us consider a real wavelet multiresolution $\left\{V_{n}\right\}_{n}^{\infty}$ in $L_{2}([a, b])$ as defined in (2). Throughout the remainder of the paper, we denote the wavelet approximation of a vector of $R$ functions $y(t) \quad\left(y_{1}(t), \ldots, y_{R}(t)\right)^{\prime}$ as follows:

$$
\Pi_{V_{n}}(y)(t) \quad \sum_{k \in \mathbb{Z}} \theta_{n, k}^{R} \phi_{n, k}(t)
$$


where $\theta_{n, k}^{R} \in \mathbb{R}^{R}$ is a vector of coefficients and $\phi$ is a scaling function. Daubechies wavelets are a good candidate because of their orthogonality, smoothness, compact support and their large number of vanishing moments. Then, a finite set of functions $\phi_{n, k}$ is only needed to approximate $y(t)$ for any $t$ (in particular we consider those functions $\phi_{n, k}$ whose support contains $t$ ) and, for the domain $[a, b]$, the number of coefficients $\left\{\theta_{n, k}^{R}\right\}$ is $2^{n} b \quad 2^{n} a+2 N \quad 2$, using Daubechies wavelets of order $N$ and a multiresolution level of $n$. (Recall that support $\phi_{n, k} \quad\left[2^{n} k, 2^{n}(k+2 N \quad 1)\right]$ and the nonzero terms in the summation in Eq. (6) ranges from $2^{n} a+2 \quad 2 N$ to $2^{n} b \quad 1$ ). In practice, we may save some computation time using a change of variables to shorten the domain $[a, b]$ in (1).

Rather than solving (1) as a standard wavelet collocation approach would do (see Judd, 1998, pp. 378 384), we are content with a finite difference approximation. Our wavelet collocation approach owes its efficiency to the manner in which the $B V P$ is finite difference approximated. For this reason, we propose the trapezoidal approach

$$
y_{n}\left(t_{i}\right) \quad y_{n}\left(t_{i-1}\right) \quad \frac{h_{n}}{2}\left(f\left(t_{i}, y_{n}\left(t_{i}\right)\right)+f\left(t_{i-1}, y_{n}\left(t_{i-1}\right)\right)\right),
$$

where $h_{n} \quad\left(t_{i} \quad t_{i-1}\right)$ (see Judd, 1998, p. 344). This is an implicit single step method, commonly used for stiff problems due to its good stability properties and its applicability to systems without high order differentiability requirements. Note also, that the computation of the derivatives $D y_{n}$ is not required (i.e. the computation of $D \phi_{n, k}$ ), reducing the computational costs with the algorithm in terms of function evaluations. Thus using Eq. (6) in the finite difference approximation (7), our problem is to solve the following system of equations in $\theta_{n, k}^{R} \in \mathbb{R}^{R}$ :

$$
\begin{aligned}
& \sum_{k \in \mathbb{Z}} \theta_{n, k}^{R}\left(\phi_{n, k}\left(t_{i, n}\right) \quad \phi_{n, k}\left(t_{i-1, n}\right)\right) \\
& \quad \frac{h_{n}}{2}\left(f\left(t_{i, n}, \sum_{k \in \mathbb{Z}} \theta_{n, k}^{R} \phi_{n, k}\left(t_{i, n}\right)\right)+f\left(t_{i-1, n}, \sum_{k \in \mathbb{Z}} \theta_{n, k}^{R} \phi_{n, k}\left(t_{i-1, n}\right)\right)\right), \\
& \sum_{k \in \mathbb{Z}} \theta_{n, k}^{R} \alpha\left(\phi_{n, k}\right) \quad c,
\end{aligned}
$$

at the points $t_{i, n} \quad 2^{-n} i$ for $i \in \mathbb{Z}$, taking values in $[a, b]$ (i.e. $t_{i, n} \quad 2^{-n} i$ for all $\left.i \in\left\{2^{n} a, \ldots, 2^{n} b\right\}\right)$. There are $2^{n} b \quad 2^{n} a+1$ equations and $2^{n} b \quad 2^{n} a+2 N \quad 2$ unknowns. The coefficients $\left\{\theta_{n, k}^{R}\right\}$ should be determined such that the residual is minimized. The solution coefficients $\left\{\theta_{n, k}^{* R}\right\}$ determine an approximated solution to the $B V P$ given by

$$
y_{n}^{*}(t) \quad \sum_{k \in \mathbb{Z}} \theta_{n, k}^{* R} \phi_{n, k}(t) .
$$

We have implemented the algorithm using MATLAB 6.0 on an Inter Centrino Pentium M $1.6 \mathrm{GHz}$ with machine precision $10^{-16}$. In Appendix A we present a MATLAB code to solve the stiff problem $\dot{y}+y \quad 0, y(0) \quad 1$, that illustrates how to compute the solution of a simple differential problem using the proposed method. 
Next a numerical experiment is introduced to show the performance of the proposed approach.

Example 1. A two body problem.

Consider the periodic problem

$$
\begin{array}{cccccc}
\ddot{y} & \frac{y}{\sqrt{y^{2}+z^{2}}}, & y(0) & 1, & \dot{y}(0) & 0, \\
\ddot{z} & \frac{z}{\sqrt{ } y^{2}+z^{2}}, & z(0) & 0, & \dot{z}(0) & 1,
\end{array}
$$

whose analytical exact solution is given by $x^{*} \quad\left(y^{*}, z^{*}\right)$ with $y^{*}(t) \cos t$ and $z^{*}(t) \quad \sin t$. We rewrite Problem (9) as

$$
\begin{array}{llllllll}
\dot{y} & u, & \dot{u} & \frac{y}{\sqrt{y^{2}+z^{2}}}, & y(0) & 1, & u(0) & 0, \\
\dot{z} & v, & \dot{v} & \frac{z}{\sqrt{y^{2}+z^{2}}}, & z(0) & 1, & v(0) & 0 .
\end{array}
$$

$x_{n}^{*} \quad\left(y_{n}^{*}, u_{n}^{*}, z_{n}^{*}, v_{n}^{*}\right)$ denotes the exact solution of (10). We approximate $x_{n}^{*}$ using Daubechies wavelets of order $N \quad 3$ and at a resolution level of $n \quad 2$. Then, we consider the system of equations (8) for (10). This is a system of nonlinear equations that is solved to optimality by the subroutine lsqnonlin corresponding to the optimization toolbox (which is a MATLAB subroutine to solve nonlinear least squares problems). The approximate solution $\left(y_{n}^{*}, z_{n}^{*}\right)$ of (9) is shown in Figs. 3 and 4 . The approximation error obtained is $\left\|y^{*}\left(t_{i, n}\right) \quad y_{\theta^{*}, n}\left(t_{i, n}\right)\right\|_{\infty} \quad 4.3 \times 10^{-3}$ and $\left\|z^{*}\left(t_{i, n}\right) \quad z_{\theta^{*}, n}\left(t_{i, n}\right)\right\|_{\infty} \quad 2.8 \times 10^{-3}$.

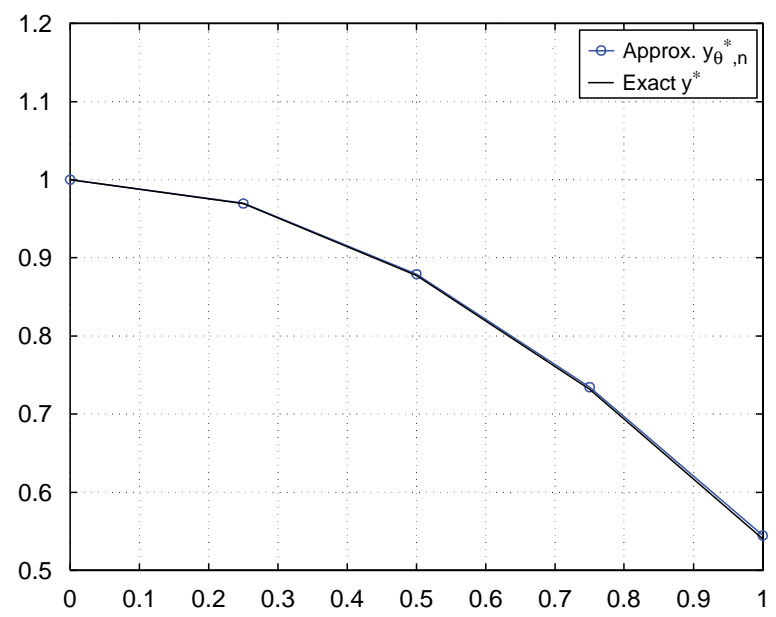

Fig. 3. Approximate solution $y_{\theta^{*}, n}$ of (9) with $N=3$ and $n=2$. 


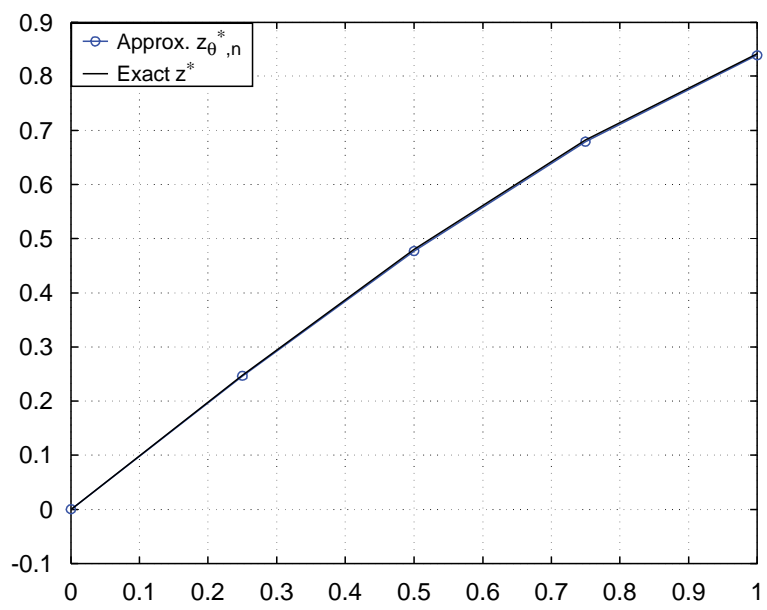

Fig. 4. Approximate solution $u_{\theta^{*}, n}$ of (9) with $N=3$ and $n=2$.

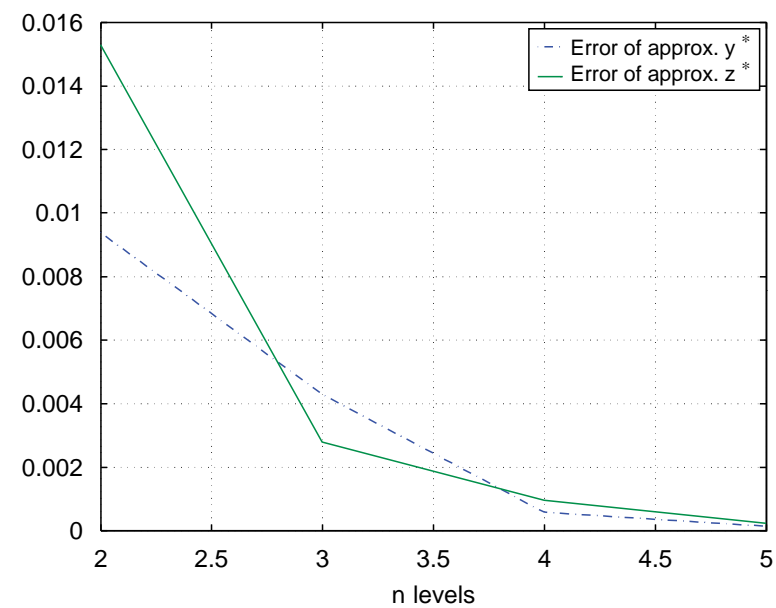

Fig. 5. Error approximation versus number of coefficients for fixed $N=2$ and different levels $n$.

Increasing the resolution level $n$ improves the accuracy of the solution. Fig. 5 shows the approximation error $\left\|y^{*}\left(t_{i, n}\right) \quad y_{\theta^{*}, n}\left(t_{i, n}\right)\right\|_{\infty}$ and $\left\|z^{*}\left(t_{i, n}\right) \quad z_{\theta^{*}, n}\left(t_{i, n}\right)\right\|_{\infty}$ versus the number of unknowns for a fixed $N \quad 2$ and multiresolution levels 


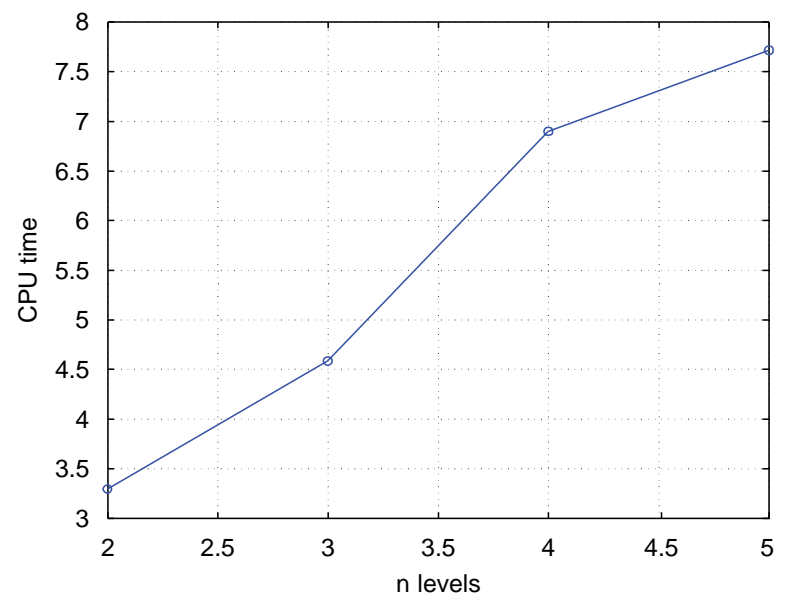

Fig. 6. Running times (in log scale) for (9) for fixed $N=2$ and different levels $n$.

$n \quad 2,3,4,5$. We will prove in Section 4 that the approximation error is $\mathrm{O}\left(2^{-n}\right)$. The parameter $N$ makes little impact on the approximation accuracy, although it can be expected to be a better fit for higher values of $N$ when the solution path exhibits stronger (smooth) fluctuations. Fig. 6 shows the running time (seconds in logarithmic scale) to obtain the approximate solution for levels $n$ 2, 3,4,5. When examining accuracy and computing time together, the choice of moderate order $N$ and level $n$ is favoured.

Daubechies wavelets constitute a basis on the entire real line, instead of on a finite interval. When such bases are used for problems on a finite interval of the real line, large errors may be encountered in the border of the interval which is not determined by any boundary condition. Fig. 7 shows the error distribution of the approximate solutions. When the results are not accurate enough, wavelet bases on a finite interval should be considered (see Daubechies, 1994).

A similar approximation error is obtained using other orthogonal wavelet bases, whose scaling functions have similar properties of smoothness, compact support and number of vanishing moments to Daubechies. Examples of this type are symlets or coiflets. We limit our study to Daubechies wavelets as the additional properties of these other wavelets does not really concern our problem.

\subsection{Continuous time life cycle consumption models}

In this section we use the proposed algorithm to solve two continuous time life cycle models to illustrate our approach. 


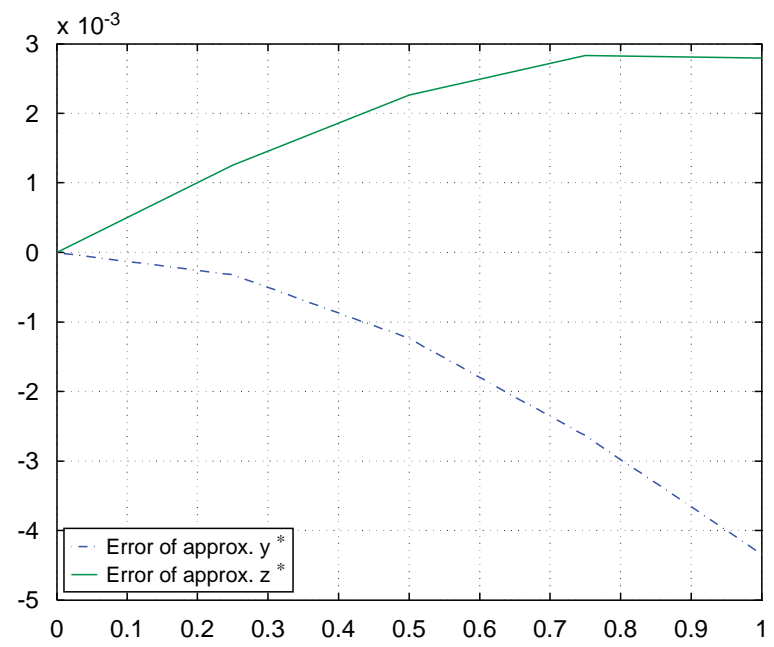

Fig. 7. Error distribution of the approximate solution of (9) with $N=3$ and $n=2$.

\subsection{Solving a continuous time life cycle problem}

Consider the continuous time life cycle model for an economy with one good and one capital stock per capita (Judd, 1998, pp. 389 392):

$$
\begin{aligned}
\max & \int_{0}^{T} \mathrm{e}^{-\rho t} u\left(c_{t}\right) \mathrm{d} t \\
\text { s.t. } & \dot{A}_{t} \quad f\left(A_{t}\right)+w_{t} \quad c_{t}, \\
& A(0) \quad a_{0}, \quad A(T) \quad a_{T} .
\end{aligned}
$$

Assume that the asset return function is given by $f(A) \quad r A$, with $r \quad 0.10 ; u(c)$

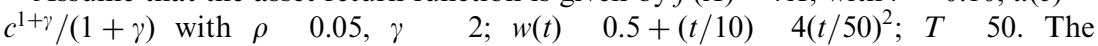
solutions $(c(t), A(t))$ of this problem are characterized by the $B V P$

$\dot{c}_{t} \quad 0.025 c_{t} \quad 0$,

$\dot{A}_{t} \quad 0.1 A_{t}+c_{t} \quad 0.5+(t / 10) \quad 4(t / 50)^{2}$,

$A(0) \quad A(50) \quad 0$.

$c^{*}(t)$ and $A^{*}(t)$ denote the true solution to (11).

First, we consider a change of the time scale to reduce the domain support from $[0,50]$ to $[0,5]$ and we approximate both consumption and asset return with Daubechies wavelets of order $N \quad 3$ and at a resolution of $n \quad 2$ :

$$
c(t) \quad \sum_{2-2 \cdot 3}^{2^{2} 5-1} \theta_{k}^{c} \phi_{2, k}(t), \quad A(t) \quad \sum_{k=2 \cdot 3}^{2^{2} 5-1} \theta_{k}^{A} \phi_{2, k}(t) .
$$


Then, we obtain the coefficients of the approximate solution by solving the following system of linear equations:

$$
\begin{aligned}
& \sum_{k}^{2-2 \cdot 3} \theta_{k}^{c}\left(\phi_{2, k}\left(t_{i}\right) \quad \phi_{2, k}\left(t_{i-1}\right)\right) \quad \frac{h_{n}}{2} 0.025 \sum_{k}^{2-2 \cdot 3} \theta_{k}^{2}\left(\phi_{2, k}\left(t_{i}\right)+\phi_{2, k}\left(t_{i-1}\right)\right) \quad 0, \\
& \sum_{k}^{2^{2} 5-1} \theta_{k}^{A}\left(\phi_{2, k}\left(t_{i}\right) \quad \phi_{2, k}\left(t_{i-1}\right)\right) \quad \frac{h_{n}}{2} 0.1 \sum_{k}^{25-1} \theta_{2-3}^{A}\left(\phi_{2, k}\left(t_{i}\right)+\phi_{2, k}\left(t_{i-1}\right)\right) \\
& 0.5 \frac{h_{n}}{2}(1+1) \quad \frac{h_{n}}{2}\left(t_{i}+t_{i-1}\right)+4 \frac{h_{n}}{2} \frac{\left(t_{i}+t_{i-1}\right)^{2}}{25} \\
& +\sum_{k}^{2^{2} 5-1} \theta_{k}^{c}\left(\phi_{2, k}\left(t_{i}\right) \quad \phi_{2, k}\left(t_{i-1}\right)\right) \quad 0, \\
& \sum_{2-2 \cdot 3}^{2^{2} 5-1} \theta_{k}^{A} \phi_{2, k}(0) \quad 0 \text {, } \\
& \sum_{2-2 \cdot 3}^{2^{2} 5-1} \theta_{k}^{A} \phi_{2, k}(5) \quad 0
\end{aligned}
$$

for all $t_{i} \quad 2^{-2} i$ with $i \quad 0, \ldots, 20$ (as $t_{i} \in[0,5]$ ). The approximate solution $\left(c_{\theta^{c *}, n}, A_{\theta^{A *}, n}\right)$ and the exact solution are shown in Figs. 8 and 9.

The maximum approximation error obtained is $\left\|E^{c}\left(t_{i, n}\right)\right\|_{\infty} \| c^{*}\left(t_{i, n}\right)$ $c_{\theta^{c *, n}}\left(t_{i, n}\right) \|_{\infty} \quad 1.6 \times 10^{-3}$ and $\left\|E^{A}\left(t_{i, n}\right)\right\|_{\infty} \quad\left\|A^{*}\left(t_{i, n}\right) \quad A_{\theta^{A *}, n}\left(t_{i, n}\right)\right\|_{\infty} \quad 3.7 \times 10^{-4}$ and the error distribution is given in Fig. 10. The maximum relative error for

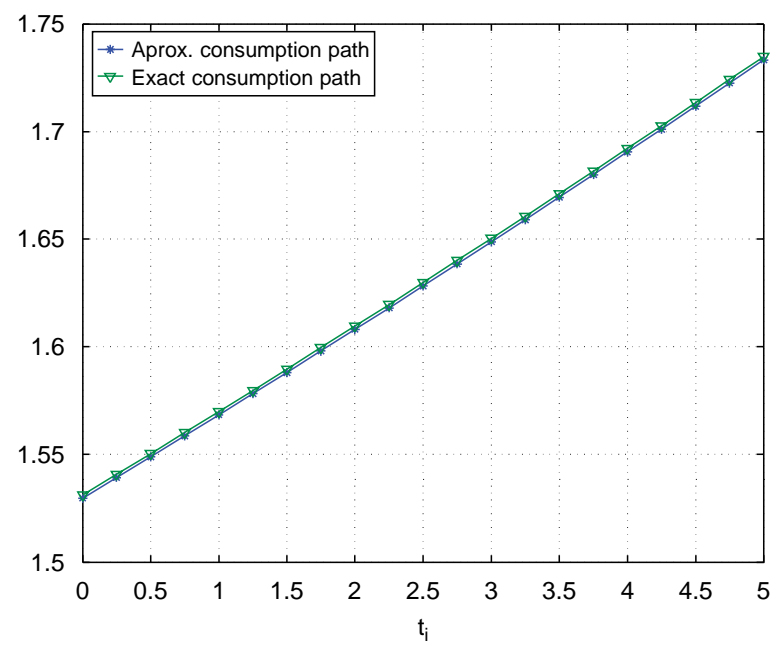

Fig. 8. Approximate solution $c_{\theta}^{c *, n}$ of (11) with $N=3$ and $n=2$. 


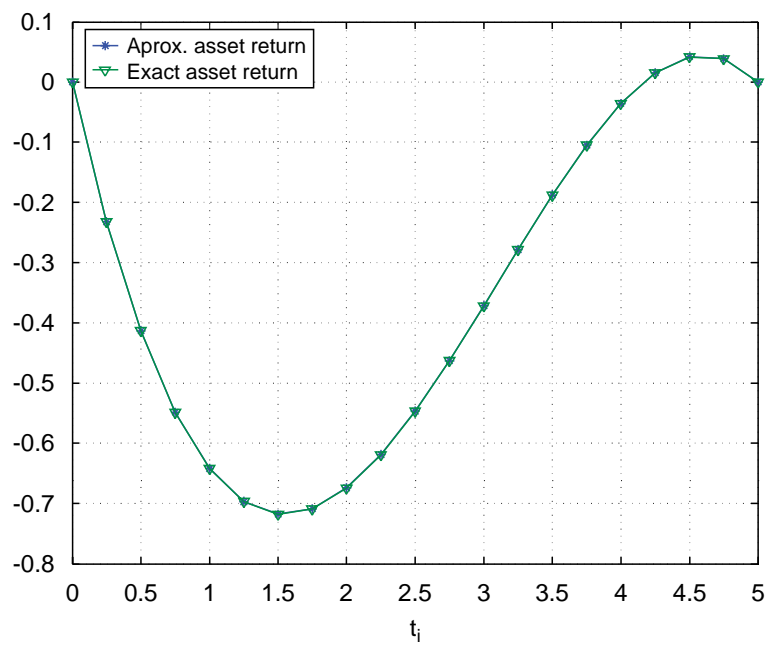

Fig. 9. Approximate solution $A_{\theta^{A *}, n}$ of (11) with $N=3$ and $n=2$.

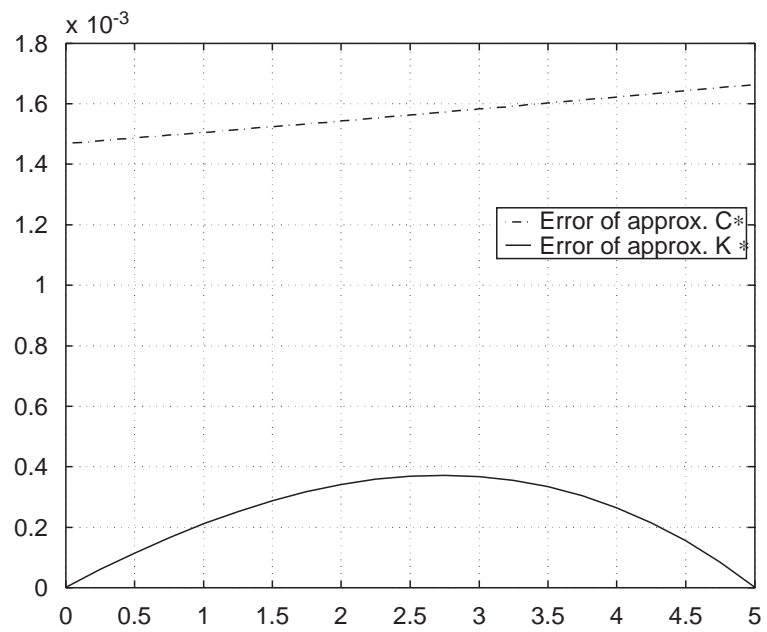

Fig. 10. Error distribution of the approximation with $N=3$ and $n=2$.

consumption and asset return defined by

$$
R E^{c}\left(t_{i, n}\right) \quad\left|\frac{c^{*}\left(t_{i, n}\right)}{c^{*}\left(t_{i, n}\right)}\right|, \quad R E^{A}\left(t_{i, n}\right) \quad\left|\frac{A^{*}\left(t_{i, n}\right)}{A^{*}\left(t_{i, n}\right)}\right| \begin{aligned}
& A^{A *, n} \\
& \left.A_{i, n}\right)
\end{aligned} \mid
$$

is shown in Fig. 11. 


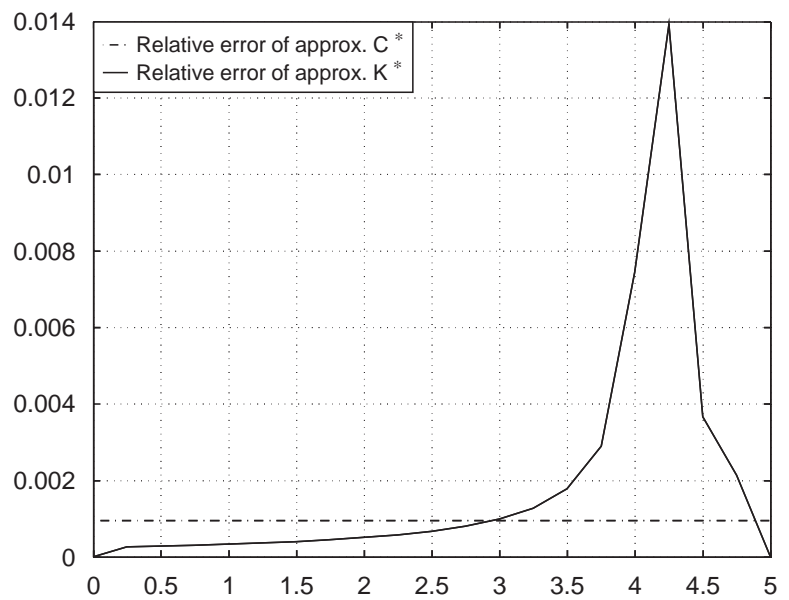

Fig. 11. Relative error distribution of approximation with $N=3$ and $n=2$.

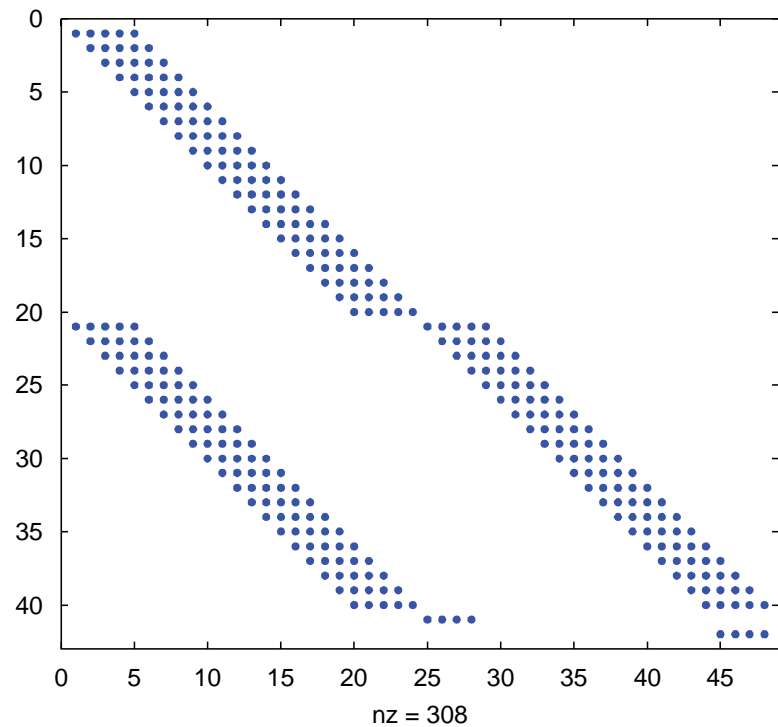

Fig. 12. The sparsity pattern of the right side of (12). The parts containing nonzero entries are shaded.

As documented in, e.g. Beylkin (1992), wavelet bases with a large number of vanishing moments (such as Daubechies) tend to enhance the sparsity of matrix operators to a greater extent. Fig. 12 plots the sparsity pattern of the matrix. This 
Maximum (absolute and relative) approximation errors and running times (seconds) until convergence for different resolution levels

\begin{tabular}{llllll}
\hline & $\left\|E^{c}\left(t_{i, n}\right)\right\|_{\infty}$ & $\left\|E^{c}\left(t_{i, n}\right)\right\|_{\infty}$ & $\left\|R E^{c}\left(t_{i, n}\right)\right\|_{\infty}$ & $\left\|R E^{A}\left(t_{i, n}\right)\right\|_{\infty}$ & CPU time \\
\hline$n=2$ & $1.6 \times 10^{3}$ & $3.7 \times 10^{4}$ & $9.5 \times 10^{4}$ & $1.3 \times 10^{2}$ & 0.08 \\
$n=3$ & $4.1 \times 10^{4}$ & $9.2 \times 10^{5}$ & $2.3 \times 10^{4}$ & $8.0 \times 10^{3}$ & 0.09 \\
$n=4$ & $1.0 \times 10^{4}$ & $2.3 \times 10^{5}$ & $5.9 \times 10^{5}$ & $3.0 \times 10^{3}$ & 0.11 \\
\hline
\end{tabular}

property is remarkable since the computing time required to solve (12) using Daubechies wavelets with $N \quad 3$ and $n \quad 2$ is $0.079 \mathrm{~s}$.

Table 1 reports the main properties of the approximate solution $\left(c_{\theta^{c *}, n}, A_{\theta^{A *}, n}\right)$ for different resolution levels $n$. From Table 1 and Figs. 1011 it can be deduced that the proposed method obtains sufficiently accurate results for practical purposes.

\subsection{A continuous time life cycle problem with borrowing constraints}

Consider the problem:

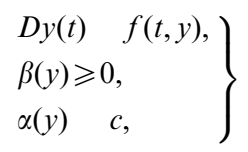

where $f$ is a continuous function, $\alpha \quad\left(\alpha_{1}, \ldots, \alpha_{R}\right)^{\prime}$ a vector of continuous linear functionals, linearly independent, and $\beta$ a continuous operator defined on $C^{1}([a, b])$. This is a $B V P$ with inequality constraints $\beta(y) \geqslant 0$. These problems typically arise as the solution of optimal control problems with inequality constraints, such as

$$
\begin{aligned}
\min & J\left(u_{t}\right) \quad \varphi\left(t_{1}, x_{t_{1}}\right)+\int_{t_{0}}^{t_{1}} \Psi\left(t, x_{t}, u_{t}\right) \mathrm{d} t \\
\text { s.t. } & \dot{x}_{t} \quad f\left(t, x_{t}, u_{t}\right), \\
& g\left(t, x_{t}, u_{t}\right) \leqslant 0, \\
& h\left(t_{1}, x_{t_{1}}\right) \leqslant 0 \\
& x_{t_{0}} \quad x_{0},
\end{aligned}
$$

see, e.g. Venkatesh (2001). These problems are used to study many questions in economics, such as multisectoral models with limitations on the intersectoral mobility of factors of production, models of inventory investment or a heterogeneous agent model with borrowing constraints.

In this section, we extend the collocation wavelets algorithm to solve $B V P$ of the type (13). Given a wavelet approximation of $y \in C^{r}(\mathbb{R})^{R}$, the inequality $\beta(y) \geqslant 0$ can be replaced by

$$
\beta\left(y_{n}\right)\left(t_{i, n}\right)+s_{i} \quad 0, \quad t_{i, n} \quad 2^{-n} i \text { for all } i \in\left\{2^{n} a, \ldots, 2^{n} b\right\},
$$


adding slack variables $s_{i} \geqslant 0$. With this approach, an approximate solution to problem (13) is given by the coefficients $\left\{\theta_{n, k}^{* R}\right\} \in \mathbb{R}^{R}$ and the slack variables $\left\{s_{i}\right\} \in \mathbb{R}^{+}$ satisfying

$$
\begin{aligned}
& \sum_{k \in \mathbb{Z}} \theta_{n, k}^{R}\left(\phi_{n, k}\left(t_{i, n}\right) \quad \phi_{n, k}\left(t_{i-1, n}\right)\right) \\
& \quad \frac{h_{n}}{2}\left(f\left(t_{i, n}, \sum_{k \in \mathbb{Z}} \theta_{n, k}^{R} \phi_{n, k}\left(t_{i, n}\right)\right)+f\left(t_{i-1, n}, \sum_{k \in \mathbb{Z}} \theta_{n, k}^{R} \phi_{n, k}\left(t_{i-1, n}\right)\right)\right), \\
& \beta\left(\sum_{k \in \mathbb{Z}} \theta_{n, k}^{R} \alpha\left(\phi_{n, k}\right)\right)\left(t_{i, n}\right)+s_{i} \quad 0, \\
& \alpha\left(\sum_{k \in \mathbb{Z}} \theta_{n, k}^{R} \phi_{n, k}\right) \quad c,
\end{aligned}
$$

with $t_{i, n} 2^{-n} i$, for all $i \in\left\{2^{n} a, \ldots, 2^{n} b\right\}$. This problem can be solved using any standard programming packages such as the MATLAB subroutine fmincon corresponding to the optimization toolbox (this routine is suited for optimization problems with nonlinear objective function and constraints).

To illustrate the implementation of this extension, consider the continuous time life cycle model for an economy with one good and one capital stock per capita and borrowing constraints:

$$
\begin{aligned}
\max & \int_{0}^{T} \mathrm{e}^{-\rho t} u\left(c_{t}\right) \mathrm{d} t \\
\text { s.t. } & \dot{A}_{t} \quad f\left(A_{t}\right)+w_{t} \quad c_{t}, \\
& A_{t} \geqslant 0, \\
& A(0) \quad a_{0}, \quad A(T) \quad a_{T} .
\end{aligned}
$$

As before, assume that the asset return function is given by $f(A) \quad r A$, with $r \quad 0.10$; the utility function $u(c) \quad c^{1+\gamma} /(1+\gamma)$ with $\rho \quad 0.05, \quad \gamma \quad 2 ; \quad w(t) \quad 0.5+$ $(t / 10) \quad 4(t / 50)^{2} ; T \quad 50$. The true solutions of this problem are denoted by $c^{*}(t)$ and $A^{*}(t)$. The solutions $(c(t), A(t))$ of this problem are characterized by

$$
\begin{aligned}
& \dot{c}_{t} \quad 0.025 c_{t} \quad 0, \\
& \begin{array}{llll}
\dot{A}_{t} & 0.1 A_{t}+c_{t} & 0.5 & (t / 10)+4(t / 50)^{2}
\end{array} \quad 0,
\end{aligned}
$$

with the boundary conditions $A(0) \quad A(50) \quad 0$ and the borrowing constraints $A_{t} \geqslant 0$, for all $t \in[0,50]$. The inequalities are equivalent to

$$
A_{t} \quad s_{t} \quad 0 \quad \text { with } s_{t} \geqslant 0 \text { for all } t \in[0,50],
$$

Furthermore, let us consider a change of the time scale to reduce the domain support from $[0,50]$ to $[0,5]$. 
We first approximate both consumption and asset return with Daubechies wavelets of order $N \quad 3$ and at a resolution of $n \quad 2$ :

$$
c(t) \quad \sum_{2-2 \cdot 3}^{2^{2} 5-1} \theta_{k}^{c} \phi_{2, k}(t), \quad A(t) \quad \sum_{k 2-2 \cdot 3}^{2^{2} 5-1} \theta_{k}^{A} \phi_{2, k}(t) .
$$

We obtain the coefficients of the approximate solution by solving the following problem:

$$
\begin{aligned}
& H^{1}(i) \quad \sum_{2-2 \cdot 3}^{2^{2} 5-1}\left(1 \quad \frac{h_{n}}{2} 0.025\right) \theta_{k}^{c}\left(\phi_{2, k}\left(t_{i}\right) \quad \phi_{2, k}\left(t_{i-1}\right)\right) \quad 0, \\
& H^{2}(i) \quad \sum_{2-2 \cdot 3}^{2^{2} 5-1}\left(1 \quad \frac{h_{n}}{2} 0.1\right) \theta_{k}^{A}\left(\phi_{2, k}\left(t_{i}\right) \quad \phi_{2, k}\left(t_{i-1}\right)\right) \\
& +\sum_{k=2 \cdot 3}^{2^{2} 5-1} \theta_{k}^{c}\left(\phi_{2, k}\left(t_{i}\right) \quad \phi_{2, k}\left(t_{i-1}\right)\right) \\
& \frac{h_{n}}{2}\left(1+\left(t_{i}+t_{i-1}\right) \quad 4 \frac{\left(t_{i}+t_{i-1}\right)^{2}}{25}\right) \quad 0, \\
& H^{3}(i) \quad \sum_{2-2 \cdot 3}^{2^{2} 5-1} \theta_{k}^{A} \phi_{2, k}\left(t_{i}\right) \quad s_{i} \quad 0 \quad \text { with } s_{i} \geqslant 0, \\
& H^{4}(i) \quad \sum_{2-2 \cdot 3}^{2^{2} 5-1} \theta_{k}^{A} \phi_{2, k}(0) \quad 0, \\
& H^{5}(i) \quad \sum_{2-2 \cdot 3}^{2^{2} 5-1} \theta_{k}^{A} \phi_{2, k}(5) \quad 0,
\end{aligned}
$$

for all $t_{i} \quad 2^{-2} i$ and for all $i \quad 0, \ldots, 20$. Problem (14) can be written with the equivalent formulation:

$$
\begin{aligned}
\min & \frac{1}{2} \sum_{j=1}^{3} \sum_{i=0}^{20} H^{j}(i)^{2}, \\
\text { s.t. } & H^{4}(i) \quad H^{5}(i) \quad 0, s_{i} \geqslant 0 \text { for all } i \quad 0, \ldots, 20 .
\end{aligned}
$$

This is a least square problem with linear inequality constraints. We use an interior point approach to solve this problem, as these methods have been proven to be efficient for bound constrained programming. In particular, we consider the algorithm presented in Esteban Bravo (2004). The approximate solution $\left(c_{\theta^{c *}, n}, A_{\theta^{A *}, n}\right)$ is shown in Figs. 13 and 14, respectively.

To make our approach comparable to standard approaches in computational economics literature, Table 2 reports the accuracy of the solution measured by the $L_{\infty}$ norm of the approximate Euler equation error; i.e. $\left\|H^{1}(i)\right\|_{\infty} \max _{i} \quad 0, \ldots, 20$ $\left|H^{1}(i)\right|$ and $\left\|H^{2}(i)\right\|_{\infty} \quad \max _{i} \quad 0, \ldots, 20\left|H^{2}(i)\right|$. As expected, the accuracy of the solution 


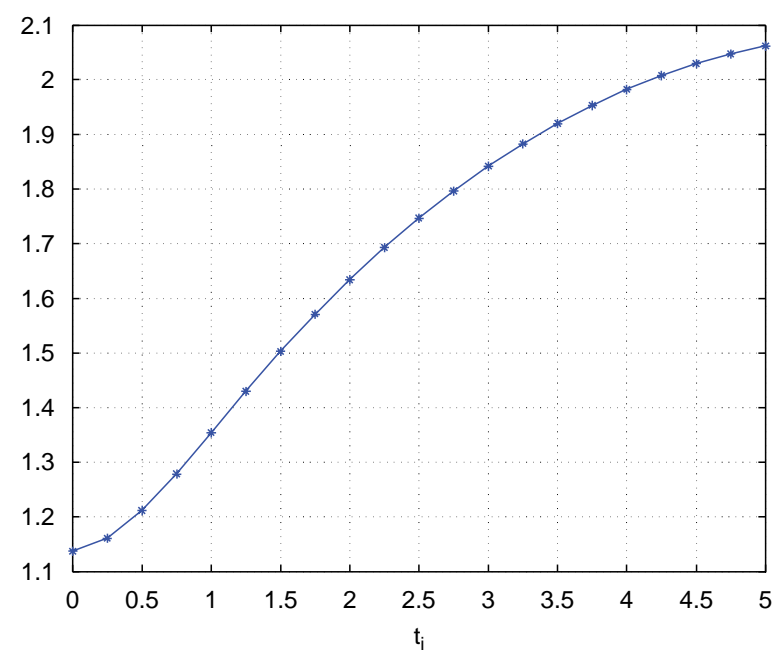

Fig. 13. Approximate solution $c_{\theta^{c *}, n}$ of (14) with $N=3$ and $n=2$.

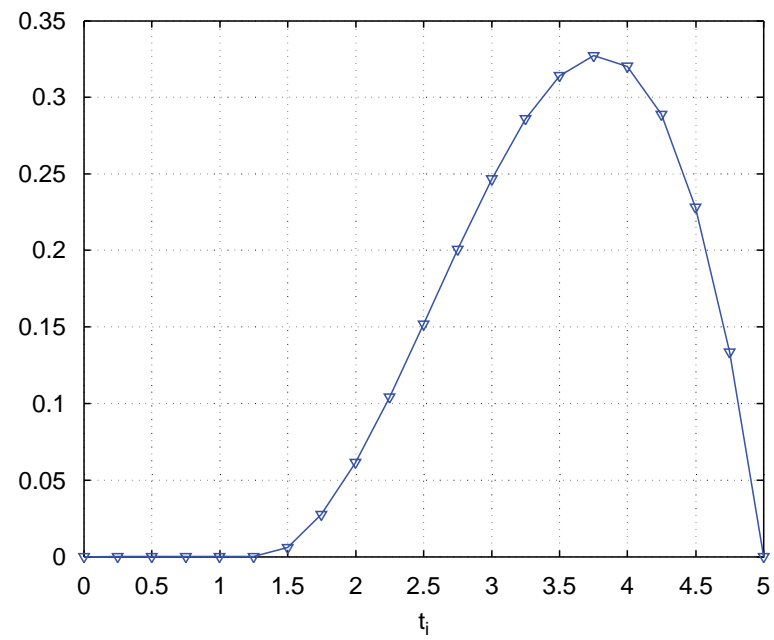

Fig. 14. Approximate solution $A_{\theta^{A *}, n}$ of (14) with $N=3$ and $n=2$.

increases with the resolution level $n$. Table 2 also reports running times until convergence for different resolution levels, being moderate in all cases.

We can see a better view of the distribution of the approximate Euler equation errors in Fig. 15, using Daubechies wavelets with $N \quad 3$ and $n \quad$ 5. From this figure 
Table 2

Maximum approximate Euler errors and running times (seconds) until convergence for different resolution levels

\begin{tabular}{llll}
\hline & $\left\|H^{1}(i)\right\|_{\infty}$ & $\left\|H^{2}(i)\right\|_{\infty}$ & CPU time \\
\hline$n=2$ & $1.6 \times 10^{2}$ & $4.3 \times 10^{2}$ & 4.34 \\
$n=3$ & $4.5 \times 10^{3}$ & $1.2 \times 10^{2}$ & 8.19 \\
$n=4$ & $1.2 \times 10^{3}$ & $3.4 \times 10^{3}$ & 17.5 \\
$n=5$ & $3.0 \times 10^{4}$ & $8.9 \times 10^{4}$ & 69.4 \\
\hline
\end{tabular}

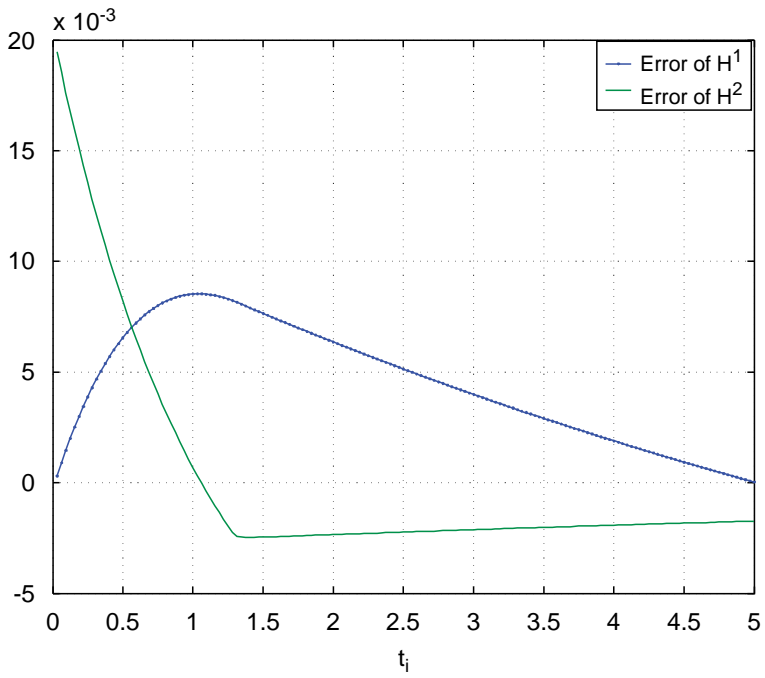

Fig. 15. Approximate Euler errors of (14) with $N=3$ and $n=5$.

and Table 2, it can be shown that the results are satisfactory in every case, although there is room for improvement in the extremes of the domain.

\section{Convergence analysis}

In this section, we will prove the convergence of the method. First, we will establish an interpolative property for wavelets. We will formulate the following assumption, which is satisfied for the commonly used wavelets:

A.1. Let $\left\{V_{n}\right\}$ be a multiresolution in $L_{2}(\mathbb{R})$, with compactly supported father wavelet $\phi$ and assume for all $y \in W_{2}^{r}(\mathbb{R})$, with $1 \leqslant r \leqslant q, q \geqslant 1$, and all inte ger $v, 0 \leqslant v \leqslant r \quad 1$, it is satisfied $\left\|D^{v} y \quad D^{v} \Pi_{V_{n}}(y)\right\|_{L_{2}} \quad \mathrm{O}\left(2^{-(r-v) n}\right)$. Whenever 
$y \in C^{r}(\mathbb{R})$ with compact support, the same rates are satisfied replacing the $L_{2}$ norm by the supremum norm. In spaces $L_{2}([a, b])$, an analogous behaviour is assumed.

There are several sufficient conditions for this result that can be found in the literature, often based on the regularity of order $q$ assumption. The father wavelet $\phi$ is said to be regular of order $q \in \mathbb{N}$, if $\phi$ has a version $q$ times continuously differentiable and for $0 \leqslant\|v\|_{1} \leqslant q$, and any positive integer $p \in \mathbb{N}$, there exists a constant $C_{p}>0$ such that $\left|D^{v} \phi(t)\right|<(1+\|t\|)^{-p} C_{p}$, for all $t \in \mathbb{R}$. See Meyer (1992) for further details. Convergence rates similar to A.1. can be also established assuming that the father wavelet has $q$ vanishing moments.

In order to prove the convergence of the proposed method, we first provide a result on interpolation which plays a crucial role in proving the wavelet collocation convergence, then we will prove the convergence of the wavelet Galerkin and finally, the convergence of the wavelet collocation methods. All the proofs can be found in Appendix B.

Theorem 2. Consider a multiresolution $\left\{V_{n}\right\}$ in $L_{2}(\mathbb{R})$ satisfying A.1. For each $y \in$ $L_{2}(\mathbb{R})$ with an a.e. continuous version with compact support, we define $\Gamma_{V_{n}}(y)$ as the function $g_{n} \in V_{n}$ such that $g_{n}\left(t_{n, i}\right) \quad y\left(t_{n, i}\right)$, for all $\left\{t_{n, i} \quad 2^{-n} i\right\}_{i \in \mathbb{Z}}$; that is, the function $g_{n}(t) \quad \sum_{k \in \mathbb{Z}} \theta_{n, k} \phi_{n, k}(t)$ satisfies

$$
\sum_{k \in \mathbb{Z}} \theta_{n, k} \phi_{n, k}\left(t_{n, i}\right) \quad y\left(t_{n, i}\right)
$$

Then, there exists a unique $\Gamma_{V_{n}}(y)$.

Furthermore, assuming

1. $\phi$ is regular of order $q \geqslant 1$, and

2. the Poisson summa $\sum_{k \in \mathbb{Z}} \Phi(\omega+2 \pi k)>0$, for almost every $\omega \in[0,2 \pi]$, with $\Phi(\omega) \quad \int_{\mathbb{R}} \phi(t) \mathrm{e}^{-\mathrm{i} t \omega} \mathrm{d} t$

for all $y \in W_{2}^{q}(\mathbb{R})$ with compact support, there exist $K>0$ and $n_{0}$ such that, $\forall n>n_{0}$,

$$
\left\|\Gamma_{V_{n}}(y) \quad y\right\|_{L_{2}} \leqslant K\left\|\Pi_{V_{n}}(y) \quad y\right\|_{W_{2}^{q}} .
$$

The same result trivially holds for multiresolutions in $L_{2}([a, b])$.

Next, we prove the convergence of the wavelet Galerkin and wavelet collocation methods. Note that there is a unique solution associated to the homogeneous problem $D y(t) \quad 0$ with $\alpha(y) \quad c$ since $\alpha$ are linearly independent (at least over $\operatorname{Ker}\{D\})$. Moreover, let us define Green's matrix of functions $G(t, z)$ such that any $g$ continuous in $[a, b]$ with $D g$ integrable can be expressed as follows:

$$
g(t) \quad P_{0}(g)(t)+\int_{a}^{b} G(t, z) D g(z) \mathrm{d} z
$$


where $P_{0}(g)$ is the unique element in $\operatorname{Ker}\{D\}$ which agrees with $\alpha(g)$. Furthermore,

$$
D g(z) \quad D_{t} P_{0}(g)(t)+\int_{a}^{b} D_{t} G(t, z) g(z) \mathrm{d} z .
$$

As a consequence, the following property can be used to express the $B V P$ in a more convenient way: let us define $D y \quad u$, thus $u \quad G[y]$ and $G^{-1}[u] y$, reciprocally, with

$$
\begin{aligned}
& G[y](t) \quad P_{0}(y)(t)+\int_{a}^{b} G(t, z) y(z) \mathrm{d} z, \\
& G^{-1}[u](t) \quad D_{t}\left\{P_{0}(y)(t)\right\}+\int_{a}^{b} D_{t} G(t, z) y(z) \mathrm{d} z .
\end{aligned}
$$

Therefore, rewriting the $B V P$ as $u \quad f\left(t, G^{-1}[u]\right)$ and defining $T(u): f\left(t, G^{-1}[u]\right)$, we can guarantee the existence of a solution in $B V P$ by proving the existence of a fixed point $u$ for $T, u \quad T u$. It is sufficient to prove that $T$ is a continuous retraction on the Banach space $C([a, b])^{R}$, and a unique solution $u_{0}$ exists, so that $y_{0} \quad G^{-1}\left(u_{0}\right)$ is the unique solution of $B V P$.

Now, given the multiresolution $\left\{V_{n}\right\}$, let $y_{n} \in V_{n}$ be the wavelet Galerkin solution to the $B V P$, and therefore $y_{n}$ satisfies

$$
\Pi_{V_{n}}\left\{D y_{n} \quad f\left(t, y_{n}\right)\right\} \quad 0, \quad \alpha\left(y_{n}\right) \quad c .
$$

The next result establishes the rate of approximation of the wavelet Galerkin method.

Theorem 3. Let us consider the problem BVP with solution $y_{0}(t)$, and a multiresolution sequence $\left\{V_{n}\right\}$ in $L_{2}([a, b])$ such that $\left\|y \quad \Pi_{V_{n}}(y)\right\|_{L_{\infty}} \rightarrow 0$, for all $y \in C([a, b])$. Let us define the curve $\mathscr{C} \quad\left\{\left(t, y_{0}(t)^{\prime}\right)^{\prime}: t \in[a, b]\right\}$. Assume that, $f \in C^{2}(\mathscr{N})$ where $\mathscr{N} \subset \mathbb{R}^{R+1}$ is an $\varepsilon$ neighbourhood of $\mathscr{C}$ in the $L_{\infty}$ norm, and it is satisfied that $\operatorname{det}\left\{\left(I \quad D_{y} f\left(t, y_{0}(t)\right)\right)\right\} \neq 0$, for all $t \in[a, b]$. Then there exist $\delta>0$ and an integer $M$ such that $y_{0}$ is unique in $B\left(y_{0}, \delta\right) \quad\left\{y:\left\|y \quad y_{0}\right\|_{L_{\infty}} \leqslant \delta\right\}$, and the projected system

$$
\Pi_{V_{n}}\left\{D y_{n} \quad f\left(t, y_{n}\right)\right\} \quad 0,
$$

has a unique solution $y_{n} \in V_{n} \cap B\left(y_{0}, \delta\right)$. Furthermore, there exists $c>0$ such that

$$
\max \left\{\left\|y_{n} \quad y_{0}\right\|_{L_{\infty}},\left\|D y_{n} \quad D y_{0}\right\|_{L_{\infty}}\right\} \leqslant c\left\|D y_{0} \quad \Pi_{V_{n}}\left(D y_{0}\right)\right\|_{L_{\infty}} .
$$

If $\left\{V_{n}\right\}$ satisfies assumption A.1. and $y \in C^{1}([a, b])$, then

$$
\max \left\{\left\|y_{n} \quad y_{0}\right\|_{L_{\infty}},\left\|D y_{n} \quad D y_{0}\right\|_{L_{\infty}}\right\} \quad \mathrm{O}\left(2^{-n}\right) .
$$

Given the multiresolution $\left\{V_{n}\right\}$, let $y_{n}^{c} \in V_{n}$ denote the wavelet collocation solution to the $B V P$, i.e. the solution to

$$
D y_{n}^{c}\left(t_{n, i}\right) \quad f\left(t_{n, i}, y_{n}^{c}\right) \quad 0, \quad \alpha\left(y_{n}^{c}\right) \quad c .
$$

The following result is an immediate consequence of Theorems 2 and 3.

Corollary 4. Under the assumptions of Theorems 2 and 3, the wavelet collocation method satisfies the $\left\|y_{n}^{c} \quad y_{0}\right\| \quad \mathrm{O}\left(2^{-n}\right)$. 
Therefore, we only need to prove the consistence of the proposed method based on the trapezoidal rule:

$$
\widetilde{y}_{n}\left(t_{i, n}\right) \quad \widetilde{y}_{n}\left(t_{i-1, n}\right) \quad \frac{h_{n}}{2}\left(f\left(t_{i, n}, \widetilde{y}_{n}\left(t_{i, n}\right)\right)+f\left(t_{i-1, n}, \widetilde{y}_{n}\left(t_{i-1, n}\right)\right)\right),
$$

for $\tilde{y}_{n} \in V_{n}$.

Proposition 5. Under the assumptions of Theorems 2 and 3. Let $\widetilde{y}_{n} \in V_{n}$ denote the approximation generated by the proposed method and $y_{n}$ the solution of the wavelet collocation method. Then, it is satisfied $\left\|\widetilde{y}_{n} \quad y_{n}^{c}\right\| \quad \mathrm{O}\left(2^{-n}\right)$ and therefore $\left\|\widetilde{y}_{n} \quad y_{0}\right\| \quad \mathrm{O}\left(2^{-n}\right)$.

Therefore, using the Daubechies wavelets at resolution $n$, the higher the value of $n$ the smaller the approximate solution error as Fig. 5 shows.

\section{Conclusions}

There are many economic problems with a boundary value structure. The finite horizon life cycle model and its extensions are relevant examples. In this paper we propose a type of collocation wavelets approach for computing $B V P$. Given a wavelets approximation, we use the trapezoidal discretization of the $B V P$ to approximate the solution of the $B V P$. The numerical results have revealed the good performance of this methodology in terms of computing time and accuracy, concluding that the proposed algorithm is promising for application in many economic problems with similar structure. Also, we have shown that the proposed approach works very well when solving $B V P$ with inequality constraints.

\section{Acknowledgment}

We thank Prof. K. Judd, Prof. B. Rustem and an anonymous referee for their constructive comments that have led to an improved version of this paper. This research has been supported by two Marie Curie Fellowships of the European Community programme IHP under contract numbers HPMF CT 200000781 and HPMF CT 200000449.

\section{Appendix A. MATLAB code example}

This MATLAB program computes the solution of the problem $\mathrm{d} y(x) / \mathrm{d} x+$ $A y(x) \quad 0$, and the initial condition $y(0) \quad 1$, for all $x, T 1<\quad x<T 2$, by the proposed wavelet based method using the implicit trapezoidal method. Solution $y \exp (x)$.

y0 1;

$\mathrm{N} \quad 3 ; \% \mathrm{~N}$ order of the Daubechies wavelets.

$\mathrm{n} \quad 2 ; \% \mathrm{n}$ level of resolution of the wavelets 
T1 $0 ; \%[\mathrm{~T} 1, \mathrm{~T} 2]$ interval

T2 6;

wname 'db3'; \% wavelet function: Daubechies, $\mathrm{dbN}$ with $\mathrm{N} \quad 3$

$\mathrm{h} \quad 2^{\wedge}(\mathrm{n}) ; \%$ distant between points where the wavelet function is defined $\%$ definition of the wavelet and scaling function given by the Wavelet toolbox [phi,psi,x] WAVEFUN(wname,n);

dimension length(x);

$\%$ number of points where the wavelet and scaling function is defined n_vars T2*2^n $1\left(\mathrm{~T}_{1} * 2 \wedge \mathrm{n}+22 * \mathrm{~N}\right)+1 ; \%$ number of coefficients theta zeros(n_vars, 1$) ; \%$ initialization of the coefficients to zero $\%$ Computing the value of $\operatorname{sum}(\mathrm{phi}(\mathrm{x}(\mathrm{i}, \mathrm{n})))$ in $\mathrm{k}$

for i $\mathrm{T} 1 * 2 \wedge \mathrm{n}+22^{*} \mathrm{~N}: \mathrm{T} 2 * 2 \wedge \mathrm{n} 1$,

$\operatorname{summ}(:, \mathrm{i}(\mathrm{T} 1 * 2 \wedge \mathrm{n}+22 * \mathrm{~N})+1) \quad$ zeros(dimension, 1$)$;

for 1 :length $(\mathrm{x})$,

phi_i(l) $\quad 0$;

if $\left(\left(\left(2^{\wedge} n\right)^{*} x(1) i>0\right) \&\left(\left(\left(2^{\wedge} n\right)\right)^{*} x(1) i<\quad 2^{*} N 1\right)\right)$,

phi_i (1) $\quad\left(2^{\wedge}(n / 2)\right) * \operatorname{phi}\left(\left(\left(2^{\wedge} n\right)^{*} x(1) i\right)^{*} 2^{\wedge} n+1\right)$;

end

end

$\operatorname{summ}\left(:, \mathrm{i}\left(\mathrm{T} 1 * 2 \wedge n+22^{*} \mathrm{~N}\right)+1\right) \quad$ phi_i(:);

end

$\%$ Compute $\operatorname{sum}(\operatorname{phi}(\mathrm{x} 0(\mathrm{i}, \mathrm{n})))$ in $\mathrm{k}$

aux_K zeros(n_vars, 1 );

for $\mathrm{j} \quad \mathrm{T} 1 * 2 \wedge n+22 * \mathrm{~N}: \mathrm{T}^{*} * 2 \wedge \mathrm{n} 1$,

if $((\mathrm{j}>0) \&(\mathrm{j}<2 * \mathrm{~N} 1))$,

aux_K(j $\left.\left(\mathrm{T} 1{ }^{*} 2^{\wedge} \mathrm{n}+22^{*} \mathrm{~N}\right)+1\right) \quad\left(2^{\wedge}(\mathrm{n} / 2)\right)^{*} \operatorname{phi}\left((\mathrm{j}) * 2^{\wedge} \mathrm{n}+1\right)$; end

end

$\%$ Compute $\mathrm{K}$ and $\mathrm{b}$ such that $\mathrm{K}$ *theta $\mathrm{b}$

$\mathrm{K} \quad[$

$(1+(\mathrm{h} / 2)) \cdot{ }^{*} \operatorname{summ}(2:$ dimension, $:)+(1+(\mathrm{h} / 2)) \cdot{ }^{*} \operatorname{summ}(1:$ dimension $1,:)$;

aux_K'];

b [zeros(dimension 1,1);y0];

$\%$ Solve the system $\mathrm{K}^{*}$ theta

b.

theta $\mathrm{K} \backslash \mathrm{b}$;

$\%$ Then, compute the approximation

x $0:$ h:T2;

aprox zeros(length(x),1);

for $\mathrm{k} T 1^{*} 2^{\wedge} \mathrm{n}+22 * \mathrm{~N}: \mathrm{T} 2 * 2 \wedge \mathrm{n} 1$,

for i 1 :dimension,

$\operatorname{phijk}(\mathrm{i}, \mathrm{k}(\mathrm{T} 1 * 2 \wedge \mathrm{n}+22 * \mathrm{~N})+1) \quad 0$;

if $\left(\left(\left(2^{\wedge} \mathrm{n}\right) * \mathrm{x}(\mathrm{i}) \mathrm{k}>\quad 0\right) \&\left(\left(\left(2^{\wedge} \mathrm{n}\right)\right)^{*} \mathrm{x}(\mathrm{i}) \mathrm{k}<\quad 2^{*} \mathrm{~N} 1\right)\right)$,

$\operatorname{phijk}\left(\mathrm{i}, \mathrm{k}\left(\mathrm{T} 1^{*} 2^{\wedge} \mathrm{n}+22^{*} \mathrm{~N}\right)+1\right) \quad\left(2^{\wedge}(\mathrm{n} / 2)\right)^{*} \operatorname{phi}\left(\left(\left(2^{\wedge} \mathrm{n}\right) * \mathrm{x}(\mathrm{i}) \mathrm{k}\right)^{*} 2^{\wedge} \mathrm{n}+1\right)$;

end

$\operatorname{aprox}\left(x(\mathrm{i}) * 2^{\wedge} \mathrm{n}+1\right) \quad \operatorname{aprox}\left(\mathrm{x}(\mathrm{i}) * 2^{\wedge} \mathrm{n}+1\right)+\operatorname{theta}\left(\mathrm{k}\left(\mathrm{T} 1 * 2^{\wedge} \mathrm{n}+22^{*} \mathrm{~N}\right)+1\right)$.

*phijk(i,k $\left.\left(\mathrm{T} 1 * 2 \wedge n+22^{*} \mathrm{~N}\right)+1\right)$; 
end

end

$\%$ Plot of the approximation and the exact solution.

f $(y 0){ }^{*} \exp (\mathrm{x})$;

figure(1)

plot(x,f, *', x,aprox,'s ')

h title('Exactand Approximate solution to dy $/ \mathrm{dx}+\mathrm{y} \quad$ 0');

h legend('Solution','Approx.',2);

xlabel(' $0 \backslash$ leq $x \backslash$ leq 6')

$\operatorname{disp}($ ("Solution Aprox $\mid$ ')

$\operatorname{disp}\left(\operatorname{norm}\left((\mathrm{y} 0) . * \exp (\mathrm{x})^{\prime}\right.\right.$ aprox $\left.)\right)$

disp('x, Solution, Approximation, Error')

$\underline{\operatorname{disp}\left(\left[\mathrm{x}^{\prime}, \mathrm{f}^{\prime}, \text { aprox, f' aprox }\right]\right)}$

\section{Appendix B. Proofs}

\section{B.1. Proof of Theorem 2}

The problem of interpolation in $V_{n}$ at points $t_{n, i} \quad 2^{-n} i$ can be reduced to solve the problem $g_{0}(i) \quad y\left(t_{n, i}\right)$ in $g_{0} \in V_{0}$ and then take $g_{n}(t) \quad 2^{n / 2} g_{0}\left(2^{n} t\right)$. Therefore, assume that $g_{0}(t) \quad \sum_{k \in \mathbb{Z}} \theta_{k} \phi(t \quad k)$ solves this problem, i.e.

$$
\sum_{k \in \mathbb{Z}} \theta_{k} \phi(i \quad k) \quad y\left(t_{n, i}\right)
$$

Clearly, a unique solution exists since $\left\{\phi\left(\begin{array}{ll}t & k\end{array}\right)\right\}_{k \in \mathbb{Z}}$ are linearly independent functions. To simplify the notation, we denote $y_{i} y\left(t_{n, i}\right)$, hence $\sum_{k \in \mathbb{Z}}$ $\theta_{k} \phi\left(\begin{array}{ll}i & k\end{array}\right) y_{i}$. This is a convolution equation that we will solve in the spectral domain. Let define the discrete Fourier transform of $\phi$ by

$$
\widetilde{\Phi}(\omega) \quad \sum_{k \in \mathbb{Z}} \phi(k) \mathrm{e}^{-\mathrm{i} k \omega} .
$$

The Poisson formula states that $\widetilde{\Phi}(\omega) \quad \sum_{k \in \mathbb{Z}} \Phi(\omega+2 \pi k)$. If $\phi$ is regular of at least order 1 , this series converges uniformly on compact sets. Furthermore, as $\widetilde{\Phi}(\omega)>0$ a.e. for $\omega \in[0,2 \pi]$, the inverse has a Fourier expansion $(1 / \widetilde{\Phi}(\omega)) \quad \sum_{k \in \mathbb{Z}} \beta_{k} \mathrm{e}^{-\mathrm{i} k \omega}$ where $b: \sum_{k \in \mathbb{Z}}\left|\beta_{k}\right|<\infty$, by the Wiener Lévy theorem. Thus, we can explicitly evaluate the coefficients $\left\{\theta_{k}\right\}$ as

$$
\theta_{k} \quad \sum_{k \in \mathbb{Z}} \beta_{k-i} y_{i} .
$$

Obviously,

$$
\sum_{k \in \mathbb{Z}}\left|\theta_{k}\right|^{2} \quad \sum_{k \in \mathbb{Z}}\left|\sum_{l \in \mathbb{Z}} \beta_{l-i} y_{i}\right|^{2} \leqslant b^{2} \sum_{k \in \mathbb{Z}}\left|y_{i}\right|^{2} \quad b^{2} \sum_{i \in \mathbb{Z}}\left|y\left(t_{n, i}\right)\right|^{2},
$$

with $\sup _{n>1} \sum_{i \in \mathbb{Z}}\left|y\left(t_{n, i}\right)\right|^{2}<\infty$ as $y$ is continuous with compact support. 
Next, we will prove that

$$
\left\|\Gamma_{V_{n}}(y)\right\|_{L_{2}} \leqslant b\|y\|_{n}
$$

where $\|y\|_{n} \quad\left(2^{-n} \sum_{i \in \mathbb{Z}}\left|y\left(t_{n, i}\right)\right|\right)^{1 / 2}$.

Notice that $\left\|g_{n}\right\|_{L_{2}} \quad 2^{-n}\left\|g_{0}\right\|_{L_{2}} \quad 2^{-n}\left\|\mathscr{F}\left(g_{0}\right)\right\|_{L_{2}}$, where $\mathscr{F}\left(g_{0}\right)(\omega)$ is the contin uous Fourier transform of $g_{0}$. We will prove that $\left\|\mathscr{F}\left(g_{0}\right)\right\|_{L_{2}}^{2} \quad \sum_{k \in \mathbb{Z}}\left|\theta_{k}\right|^{2}$ and the result follows. Let define $\widetilde{c}(\omega) \quad \sum_{k \in \mathbb{Z}} \theta_{k} \mathrm{e}^{-\mathrm{i} k \omega}$, then

$$
\begin{aligned}
\left\|\mathscr{F}\left(g_{0}\right)\right\|_{L_{2}}^{2} \quad & \int_{\mathbb{R}}\left|\mathscr{F}\left(\sum_{k \in \mathbb{Z}} \theta_{k} \phi_{0, k}\right)(\omega)\right|^{2} \mathrm{~d} \omega \quad \int_{\mathbb{R}}\left|\Phi(\omega)\left(\sum_{k \in \mathbb{Z}} \theta_{k} \mathrm{e}^{-\mathrm{i} k \omega}\right)\right|^{2} \mathrm{~d} \omega \\
& \int_{\mathbb{R}}|\Phi(\omega) \widetilde{c}(\omega)|^{2} \mathrm{~d} \omega \quad \sum_{k \in \mathbb{Z}} \int_{2 k \pi}^{2(k+1) \pi}|\Phi(\omega) \widetilde{c}(\omega)|^{2} \mathrm{~d} \omega \\
& \int_{0}^{2 \pi}|\widetilde{c}(\omega)|^{2}\left|\sum_{k \in \mathbb{Z}} \Phi(\omega+2 \pi k)\right|^{2} \mathrm{~d} \omega \\
& \int_{0}^{2 \pi}|\widetilde{c}(\omega)|^{2} \mathrm{~d} \omega \quad \sum_{k \in \mathbb{Z}}\left|\theta_{k}\right|^{2},
\end{aligned}
$$

as $\{\phi(t \quad k)\}_{k \in \mathbb{Z}}$ is orthonormal if and only if $\sum_{k \in \mathbb{Z}}|\Phi(\omega+2 \pi k)|^{2} \quad 1$ a.e., for details see Daubechies (1992). Hence, we have that $\left\|\Gamma_{V_{n}}(y)\right\|_{L_{2}}^{2} \leqslant b^{2}\|y\|_{n}^{2}$.

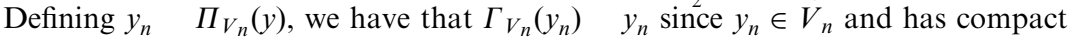
support. And as a consequence

$$
\begin{aligned}
& \left\|\Gamma_{V_{n}}(y) \quad y\right\|_{L_{2}} \quad\left\|\Gamma_{V_{n}}\left(y_{n} \quad y\right)+y_{n} \quad y\right\|_{L_{2}} \leqslant b^{2}\left\|y_{n} \quad y\right\|_{n}+\left\|y_{n} \quad y\right\|_{L_{2}} \\
& b^{2}\left\|\Pi_{V_{n}}(y) \quad y\right\|_{n}+\left\|\Pi_{V_{n}}(y) \quad y\right\|_{L_{2}} .
\end{aligned}
$$

Moreover, as for all $y \in W_{2}^{r}(\mathbb{R})$, with $r \geqslant 1$,

$$
\|y\|_{n}^{2} \leqslant C\left\{\int_{-2^{n^{n}}}^{2^{n} \pi}|\mathscr{F}(y)(\omega)|^{2} \mathrm{~d} \omega+2^{-n r}\|y\|_{W_{2}^{r}}^{2}\right\}
$$

see Thomée (1973, Lemma 4.4), the result follows applying the same bound to $\left\|\Pi_{V_{n}}(y) \quad y\right\|_{n}^{2}$.

\section{B.2. Proof of Theorem 3}

We will use the following theorem,

Theorem 6. Let $B$ a Banach space, $\left\{V_{n}\right\} \subset B$ a sequence of increasing linear subspaces, and $\Pi_{V_{n}}$ a sequence of continuous projections converging pointwise to the identity operator on B. Let $T$ a (nonlinear) operator in B. If $\left(\begin{array}{ll}1 & T\end{array}\right) u \quad 0$ has a solution $u_{0}, T$ is continuously Frechet differentiable at $u_{0}$ and $\left(\begin{array}{ll}1 & T_{u_{0}}^{\prime}\end{array}\right) u \quad 0$ has only the trivial solution in $B$, then $u_{0}$ is unique in some sphere $B\left(u_{0}, \delta\right) \quad\left\{u \in B:\left\|u \quad u_{0}\right\| \leqslant \delta\right\}$ for some $\delta>0$, and there exists an integer $M$ such that for all $n>M$ the equation $\Pi_{V_{n}}\{(1 \quad T) u\} \quad 0$ has a unique solution $u_{n} \in V_{n} \cap B\left(u_{0}, \delta\right)$. Moreover, $\exists K>0$ 
such that

$\left\|u_{n} \quad u_{0}\right\| \leqslant K\left\|\Pi_{V_{n}} u_{0} \quad u_{0}\right\|$.

Proof. See e.g. Vainikko (1967, Theorem 5).

Using the properties of the Green's function and the continuity of $f$, the functional $T$ is continuous relative to the uniform norm on a neighborhood of $u_{0} \quad G\left(y_{0}\right)$. Since (1 $T) u \quad 0$ can be seen as an equation in $C([a, b])$, we will consider the equation

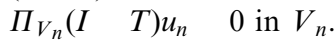

First, we check the continuously Frechet differentiability of $T$. For any $u \in$ $B\left(u_{0}, \delta\right)$ define $h \quad u \quad u_{0}$. Notice that $\mathscr{N}$ contains all line segments in $\mathbb{R}^{R+1}$ such as $\left\{u_{0}+\theta h: \theta \in[0,1]\right\}$, since

$$
y(t) \quad y_{0}(t) \quad \int_{a}^{b} D_{t} G(t, z) h(z) \mathrm{d} z,
$$

with $\left\|y \quad y_{0}\right\|_{L_{\infty}}<\varepsilon$ whenever $\delta$ is small enough, using that

$$
\chi: \quad \underset{t \in[a, b]}{\operatorname{ess} \sup } \int_{a}^{b}\left|D_{t} G(t, z)\right| \mathrm{d} z<\infty .
$$

Notice that $u_{0} \quad G\left(y_{0}\right)$. We will see that the Frechet derivative $T$ at $u_{0}(t) \quad D y_{0}(t)$ respect to the direction $h \quad\left(\begin{array}{ll}u & u_{0}\end{array}\right)$ is given by

$$
T_{u_{0}}^{\prime}(h)(t) \quad D_{u} f\left(t, u_{0}\right) \int_{a}^{b} D_{t} G(t, z) h(z) \mathrm{d} z,
$$

and the error term is given by

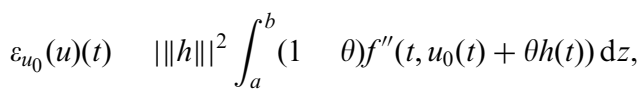

being $f^{\prime \prime}$ the second directional derivative of $f(t, \cdot)$ in the direction $h /|\|h\||$, and \|\|$h\left\|^{2} \quad \sum_{r 1}^{R}\right\| h_{r} \|^{2}$. Clearly $\left\|\varepsilon_{u_{0}}(u)\right\|_{L_{\infty}} \leqslant c_{1}\left\|u \quad u_{0}\right\|_{L_{\infty}}^{2}$, where $c_{1}$ is the maximum between the bound on $f^{\prime \prime}$ over all directions on adh $(\mathscr{N})$ and $\chi$.

Notice also that $T_{u_{0}}^{\prime}(h)(t)$ can be expressed in the original domain as the operator $T_{y_{0}}^{\prime}(y) \quad D_{y} f\left(t, y_{0}(t)\right) D y$. Since $\operatorname{det}\left\{\left(I \quad D_{y} f\left(t, y_{0}(t)\right)\right)\right\} \neq 0$, for all $t \in[a, b]$, there exists a unique trivial solution for

$$
\text { ( } \left.\quad D_{y} f\left(t, y_{0}(t)\right)\right) D y \quad 0
$$

with $\alpha(y) \quad c$. This implies the same result for $\left(\begin{array}{lll}I & T_{u_{0}}^{\prime}\end{array}\right) u \quad 0$, hence assumptions of Theorem 6 are satisfied.

Thus, there exists an integer $M>0$ such that, for all $n>M$ a solution $u_{n} \in V_{n}$ exists and is unique in the same sphere. Moreover, there exists a constant $c>0$ such that $u_{n} \quad D y_{n}, u_{0} \quad D y_{0}$ and

$$
\left\|u_{n} \quad u_{0}\right\|_{L_{\infty}} \leqslant c\left\|\Pi_{V_{n}} u_{0} \quad u_{0}\right\|_{L_{\infty}} .
$$


By the Banach Steinhaus theorem,

$$
\begin{gathered}
\left\|\Pi_{V_{n}} u_{0} \quad u_{0}\right\|_{L_{\infty}} \quad\left\|\Pi_{V_{n}}\left(u_{0} \quad u\right) \quad\left(\begin{array}{ll}
u & u_{0}
\end{array}\right)\right\|_{L_{\infty}} \quad\left\|\left(\begin{array}{lll}
1 & \Pi_{V_{n}}
\end{array}\right)\left(\begin{array}{ll}
u_{0} & u
\end{array}\right)\right\|_{L_{\infty}} \\
\leqslant c^{\prime} \inf \left\{\left\|u_{0} \quad u\right\|_{L_{\infty}}: u \in V_{n}\right\},
\end{gathered}
$$

therefore, $\exists K>0$ such that

$$
\left\|D y_{n} \quad D y_{0}\right\|_{L_{\infty}} \quad\left\|u_{n} \quad u_{0}\right\|_{L_{\infty}} \leqslant K \inf \left\{\left\|u_{0} \quad u\right\|_{L_{\infty}}: u \in V_{n}\right\} .
$$

Finally, using that $y_{n} \quad y_{0} \quad G^{-1}\left(\begin{array}{ll}u_{n} & u_{0}\end{array}\right)$, we have

$$
\left\|y_{n} \quad y_{0}\right\|_{L_{\infty}} \leqslant\left\|G^{-1}\right\|_{L_{\infty}}\left\|u_{n} \quad u_{0}\right\|_{L_{\infty}},
$$

and the rate $\mathrm{O}\left(2^{-n}\right)$ follows from Assumption A.1.

\section{B.3. Proof of Proposition 5}

By assumption $f \in C^{2}(\mathscr{N})$. The collocation solution satisfies the system $y_{n}^{c}\left(t_{i, n}\right)$ $y_{n}^{c}\left(t_{i-1, n}\right) \quad \int_{t_{i-1, n}}^{t_{i, n}} f\left(t, y_{n}^{c}(t)\right) \mathrm{d} x$, and we have proved that $y_{n}^{c} \rightarrow y_{0}$ uniformly. Using the trapezoidal integration rule it can be expressed as

$$
\begin{aligned}
y_{n}^{c}\left(t_{i, n}\right) \quad y_{n}^{c}\left(t_{i-1, n}\right) \quad \frac{h_{n}}{2}\left[f\left(t_{i, n}, y_{n}^{c}\left(t_{i}\right)\right)+f\left(t_{i-1, n}, y_{n}^{c}\left(t_{i-1, n}\right)\right)\right] \\
\\
\frac{h_{n}^{3}}{12} f^{\prime \prime}\left(\xi_{i, n}, y_{n}^{c}\left(\xi_{i, n}\right)\right),
\end{aligned}
$$

with $\xi_{i, n} \in[a, b]$. Let $A_{n}\left(y_{n}^{c}\right) \quad b_{n}\left(y_{n}^{c}\right)$ denote this system of nonlinear equations, where $\left\|b_{n}\left(y_{n}^{c}\right)\right\| \leqslant h_{n}^{3} M / 12$.

Let $\widetilde{y}_{n}(t)$ denote the solution of the proposed method that satisfies (16). Let $A_{n}\left(\widetilde{y}_{n}\right) \quad 0$ denote this system of nonlinear equations. Then, applying the mean value theorem, we have

$$
b_{n}\left(y_{n}^{c}\right) \quad A_{n}\left(y_{n}^{c}\right) \quad A_{n}\left(\widetilde{y}_{n}\right)+D A_{\varphi_{n}}\left(y_{n}^{c} \quad \tilde{y}_{n}\right) \quad D A_{\xi_{n}}\left(\begin{array}{ll}
y_{n}^{c} & \tilde{y}_{n}
\end{array}\right),
$$

where $D A_{\varphi_{n}}$ is the Frechet derivative at some intermediate point $\varphi_{n}$. Since $D A_{\varphi_{n}}(\cdot)$ has uniformly continuous inverse, it is satisfied

$$
\left\|y_{n}^{c} \quad \widetilde{y}_{n}\right\| \leqslant c h_{n}^{3} M / 12,
$$

and the result follows.

\section{References}

Ascher, U.M., Mattheij, M.M., Russell, R.D., 1995. Numerical solution of boundary value problems for ordinary differential equations. Classics in Applied Mathematics, vol. 13. Society for Industrial and Applied Mathematics, Philadelphia.

Battle, G., 1987. A block spin construction of ondelettes. Commun. Math. Phys. 110, 601615.

Beylkin, G., 1992. On the representation of operators in basis of compactly supported wavelets. SIAM J. Numer. Anal. 6, 17161740.

Beylkin, G., 1993. Wavelets and fast numerical algorithms. In: Different perspectives on wavelets. Proceedings of Symposia in Applied Mathematics, vol. 47. American Mathematical Society, RI. 
de Boor, C., Schwartz, B., 1973. Collocation at Gaussian points. SIAM J. Numer. Anal. 10, 582606. Chui, C.K. (Ed.), 1992. Wavelets. A tutorial in Theory and Applications. Academic Press, Boston, MA. Conte, S.D., 1966. The numerical solution of linear boundary value problems. SIAM Rev. 8, 309321.

Daubechies, I., 1992. Ten Lectures on Wavelets. (2nd printing with corrections) CBMS-NFS Series in Applied Mathematics. SIAM, Philadelphia, PA.

Daubechies, I., 1994. Two Recent Results on Wavelets. In: Schumaker, L.L., Webb, G. (Eds.), Recent Advances in Wavelet Analysis, Wavelet Analysis and its Applications, vol. 3. Academic Press, Boston, MA, pp. 237257.

Esteban-Bravo, M., 2004. Computing equilibria in general equilibrium models via interior-point method. Computational Economics 23 (2), 147171.

Goffe, W.L., 1993. A user's guide to the numerical solution of two-point boundary value problems arising in continuous time dynamic economic models. Computational Economics 6 (3 4), 249255.

Haar, A., 1910. Zur Theorie der orthogonalen Funktionen-Systeme. Math. Ann. 69, 331371.

Judd, K.L., 1998. Numerical Methods in Economics. The MIT Press, Cambridge, MA.

Keller, H.B., 1968. Numerical Methods for Two Point Boundary Value Problems. Ginn-Blaisdell, Waltham, MA.

Keller, H.B., 1976. Numerical Methods for Two Point Boundary Value Problems. CBMS-NSF Regional Conference Series in Applied Mathematics. Capital City Press, Montpelier, Vermont.

Latto, A., Resnikoff, H.L., Tenenbaum, E., 1991. The evaluation of connection coefficients of compactly supported wavelets. In: Maday, Y. (Ed.), Proceedings of the French-USA Workshop on Wavelets and Turbulence. Princeton University, Springer, New York.

Lemarié, P.G., 1988. Ondelettes a localisation exponentielle. J. Math. Pures Appl. 67, 227236.

Lucas, T.R., Reddien, G.W., 1972. Some collocation methods for nonlinear boundary value problems. SIAM J. Numer. Anal. 9, 341356.

Mallat, S., 1989. A theory of multiresolution signal decomposition, the wavelet representation. IEE Trans. Pattern Anal. Machine Intell. 11, 674693.

Marimon, R., Scott, A. (Eds.), 1999. Computational Methods for the Study of Dynamics Economics. Oxford University Press, Oxford.

Meyer, Y., 1992. Wavelets and Operators. Cambridge University Press, Cambridge, UK. (English translation from French, Ondelettes et opérateurs, vols. I and II, 1990, Hermann, Paris).

Meyer, Y., 1993. Wavelets: Algorithms and Applications. SIAM, Philadelphia, PA.

Osborne, M.R., 1969. On shooting methods for boundary value problems. J. Math. Anal. Appl. 27, 417433.

Prenter, P.M., 1975. Splines and Variational Methods. Wiley, New York.

Roberts, S.M., Shipman, J.S., 1972. Two-point boundary value problems: shooting methods. Modern analytic and computational methods in science and mathematics, vol. 31. American Elsevier, New York.

Russell, R.D., Shampine, L.F., 1972. A collocation method for boundary value problems. Numer. Math. 19, 128.

Rust, J., 1996. Numerical Dynamic Programming in Economics. In: Amman, H.M., Kendrick, D.A., Rust, J. (Eds.), Handbook of Computational Economics, vol. I. Elsevier Science, Amsterdam.

Santos, M., 1999. Numerical solution of dynamic economic models. In: Taylor, J.B., Woodford, M. (Eds.), Handbook of Macroeconomics, vol. I. Elsevier Science, Amsterdam.

Thomée, V., 1973. Convergence estimates for semi-discrete Galerkin methods for initial value problems. Lecture Notes in Mathematics, vol. 333, pp. 243262

Vainikko, G.M., 1967. Galerkin's perturbation method and the general theory of approximate methods for nonlinear equations. U.S.S.R. Comput. Math. and Math. Phys. 7, 141.

Varga, R.S., 1971. Functional Analysis and Approximation Theory in Numerical Analysis. Regional Conference Series in Applied Mathematics, vol. 3. SIAM, Philadelphia, PA.

Venkatesh, P.K., 2001. On the optimum control of differential-algebraic equations. J. Optim. Theory Appl. 109, 675689.

Walnut, D.F., 2001. An Introduction to Wavelets Analysis. Birkhauser, Boston. 